\title{
Emission line AGNs from the REX survey
}

\section{Results from optical spectroscopy ${ }^{\star}$}

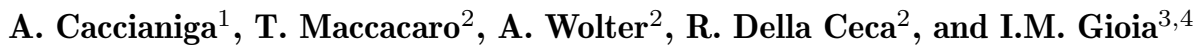 \\ 1 Observatório Astronómico de Lisboa, Tapada da Ajuda, 1349-018 Lisboa, Portugal \\ 2 Osservatorio Astronomico di Brera, via Brera 28, I-20121 Milano, Italy \\ 3 Institute for Astronomy, 2680 Woodlawn Drive, Honolulu, HI 96822, U.S.A. \\ ${ }^{4}$ Istituto di Radio Astronomia del CNR, via Gobetti 101, I-40129 Bologna, Italy
}

Received December 2, 1999; accepted March 16, 2000

\begin{abstract}
We present 71 Emission Line objects selected from the REX survey. Except for 3 of them, for which the presence of an active nucleus is dubious, all these sources are Active Galactic Nuclei (QSOs, Seyfert galaxies, emission line radiogalaxies). In addition, we present the spectra of other 19 AGNs included in a preliminary version of the REX catalog but not in the final one. The majority (80) of the 90 sources presented in this paper is newly discovered. Finally, we present the general properties in the radio and in the X-ray band of all the AGNs discovered so far in the REX survey.
\end{abstract}

Key words: galaxies: active — galaxies: seyfert galaxies: starburst - quasars: emission lines techniques: spectroscopic — surveys

\section{Introduction}

The REX survey is an effort aimed at the selection of a sizable, statistically complete sample of Radio-Emitting X-ray sources (REXs). A detailed description of this survey has been presented in Caccianiga et al. (1999). In summary, the REX survey is the result of a positional cross-correlation between the NRAO VLA Sky Survey (NVSS, Condon et al. 1998) at $1.4 \mathrm{GHz}$ and an X-ray catalog of about 17000 serendipitous sources detected in 1202 pointed ROSAT PSPC fields. The flux density limit in the radio band (at $1.4 \mathrm{GHz}$ ) is $5 \mathrm{mJy}$ while in the X-ray band the flux limits range from $\sim 3.510^{-14} \mathrm{erg} \mathrm{s}^{-1} \mathrm{~cm}^{-2}$

Send offprint requests to: A. Caccianiga,

e-mail: caccia@oal.ul.pt

* Partly based on observations collected at the European Southern Observatory, La Silla, Chile. to $\sim 210^{-13} \mathrm{erg} \mathrm{s}^{-1} \mathrm{~cm}^{-2}(0.5-2.0 \mathrm{keV})$. The area covered at the highest flux limit is about $2200 \mathrm{deg}^{2}$. The cross-correlation has produced a catalog of $\sim 1600$ REX sources. The spectroscopic observation of the REXs is in progress and, to date, the percentage of identifications is about $30 \%$. Among these sources, 232 Emission Line (EL) objects (mostly AGNs), 72 BL Lacs and 176 optically "non-active" galaxies have been found. About 30\% of these objects are newly discovered. A previous paper (Wolter et al. 1998) reported a small fraction of these new identifications; in this paper we present the spectra of 71 EL objects not included in Wolter et al. (1998). In addition, we present other 19 EL objects that were included in a preliminary version of the REX survey but not in the final catalog.

The paper is organized as follows: in Sect. 2 we present the optical observations, in Sect. 3 we explain the criteria used for the classification of the EL sources, in Sect. 4 we discuss the general radio and X-ray properties of the EL AGNs discovered so far in the REX survey. Our conclusions are summarized in Sect. 5. Throughout this paper we use $H_{0}=50 \mathrm{~km} \mathrm{~s}^{-1} \mathrm{Mpc}^{-1}$ and $q_{0}=0$.

\section{Spectroscopic observations}

Several spectroscopic observations of REX sources have been carried out during the period 1995/1998 using the $88^{\prime \prime}$ telescope of the University of Hawaii (UH) in Mauna Kea (5 observing runs), the $2.2 \mathrm{~m}$ and $3.6 \mathrm{~m}$ telescopes of ESO in La Silla (1 run) and the UNAM $2.1 \mathrm{~m}$ telescope in S. Pedro Martir (3 runs). The results coming from the first two observing runs at UNAM have been presented in Wolter et al. (1998) and will not be considered here.

The instrumental configurations are summarized in Table 1. In all cases, we have used a long-slit and low dispersion (from $3.7 \AA$ /pixel to $13.2 \AA$ /pixel) grating that 


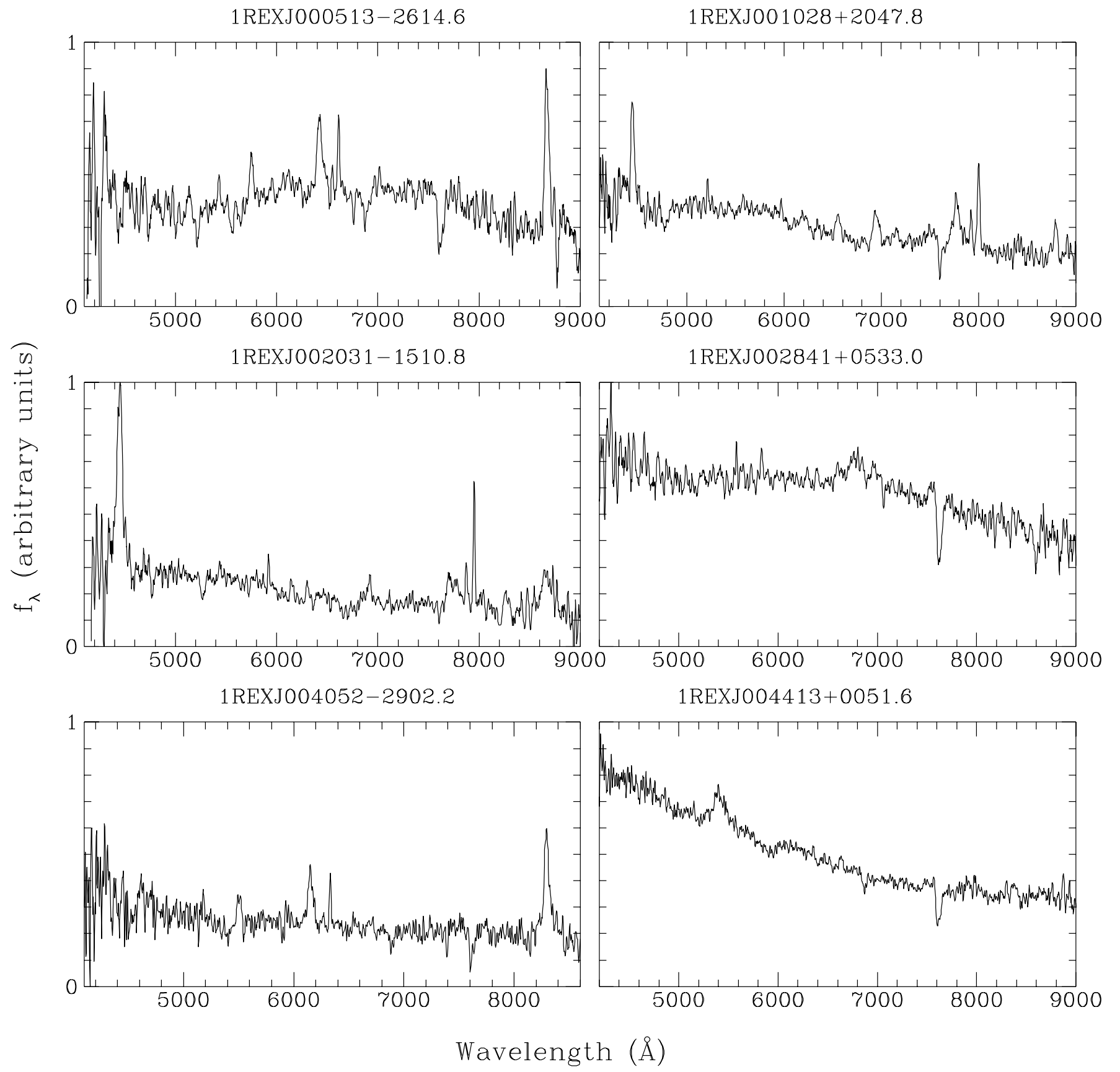

Fig. 1. Optical spectra of the 71 EL AGNs included in the REX survey

maximizes the wavelength coverage. For the data reduction we have used the IRAF longslit package. The spectra have been wavelength calibrated using an He-Ar (UNAM, $\mathrm{ESO}$ ) or a $\mathrm{Hg}-\mathrm{Cd}-\mathrm{Zn}(\mathrm{UH})$ reference spectrum. The photometric standard stars used for the relative flux calibration are: Feige 34 (UNAM 96/12), HD 19445 (UH 96/01, UH96/08), LTT377 (ESO 96/12), SAO 098781 (UH 97/03), HD 84937 (UH 98/02), PG0216+032 (UH 98/10).

In general, we have two exposures for each object, except for few cases in which we have only one spectrum.
The subtraction of the cosmic rays has been made manually, from the extracted spectrum.

On average, the seeing during the observing runs ranged from 0.9" (August 1996, December 1996, February $1998)$ to $1.5^{\prime \prime}$. We have consequently used, except for few cases, a slit of $1.5-1.6^{\prime \prime}$ to maximize the signal.

In Tables 2, 3 and 4 we present the journals of the observing runs at UH $88^{\prime \prime}$, ESO $2.2 \mathrm{~m} / 3.6 \mathrm{~m}$ and UNAM $2.1 \mathrm{~m}$ telescopes, respectively. For each object we report name, NVSS position (J2000), X-ray flux corrected for Galactic absorption (in units of $10^{-13} \mathrm{erg} \mathrm{s}^{-1} \mathrm{~cm}^{-2}$ in 
1REXJ005229-3743.8
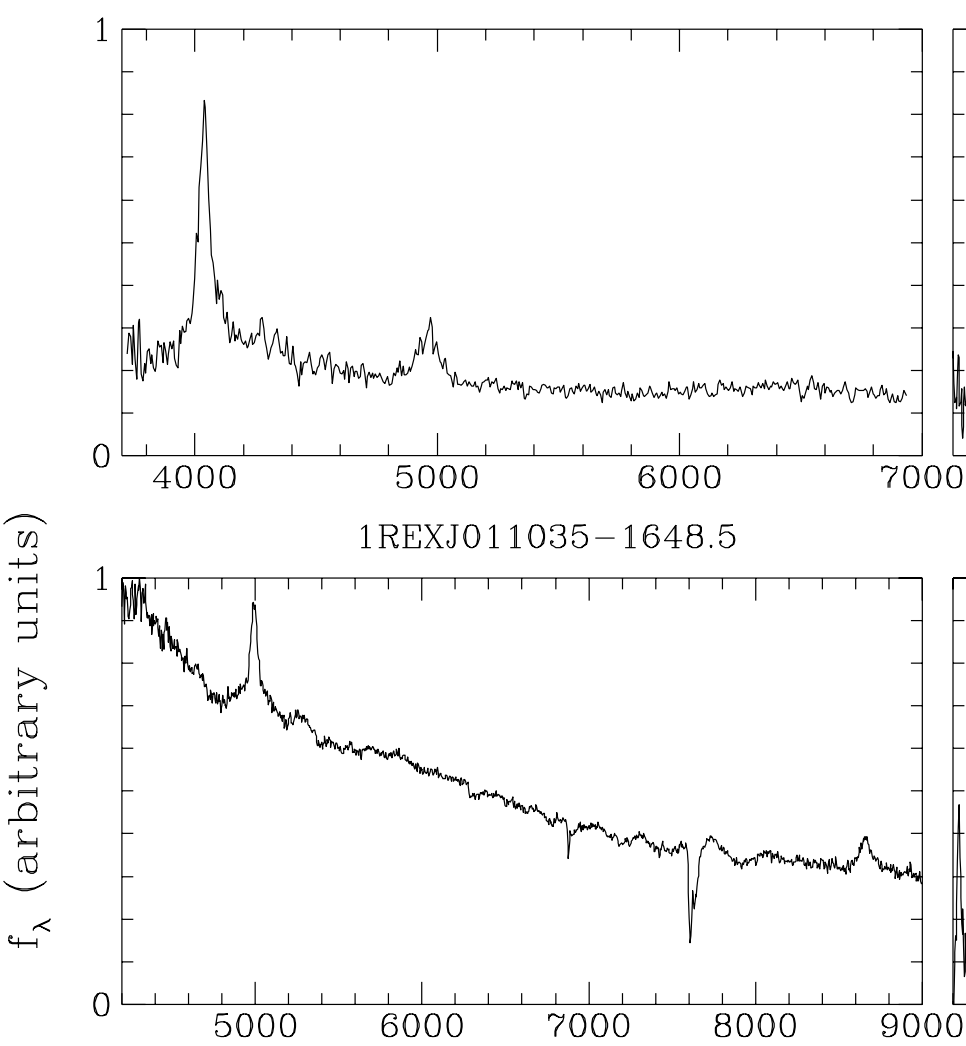

1REXJ012526+0856.5

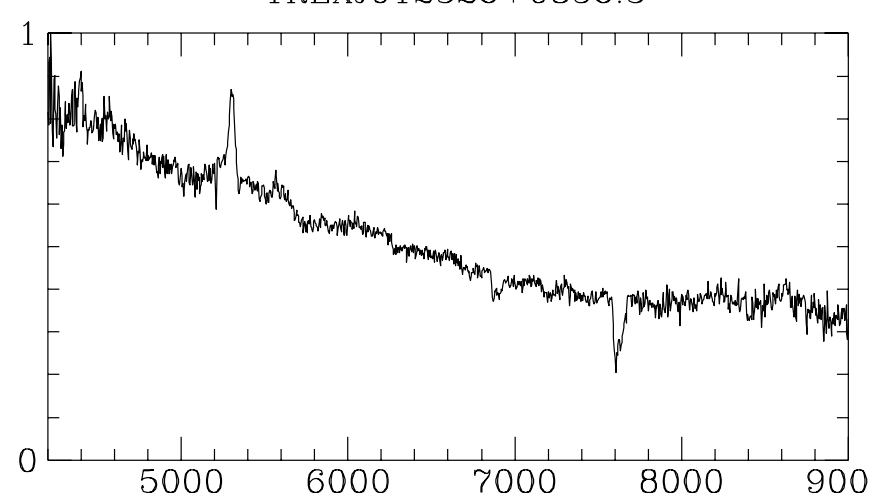

1REXJ005924+2703.5

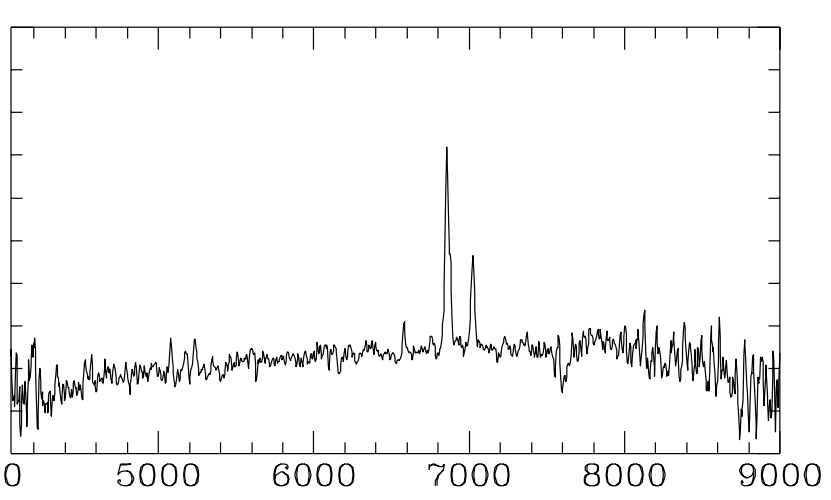

1REXJ012210+0931.7

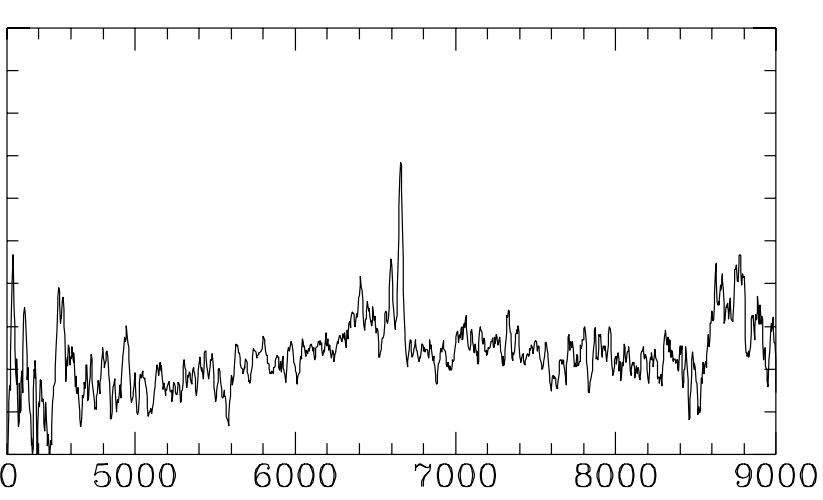

1REXJ014318+0228.3

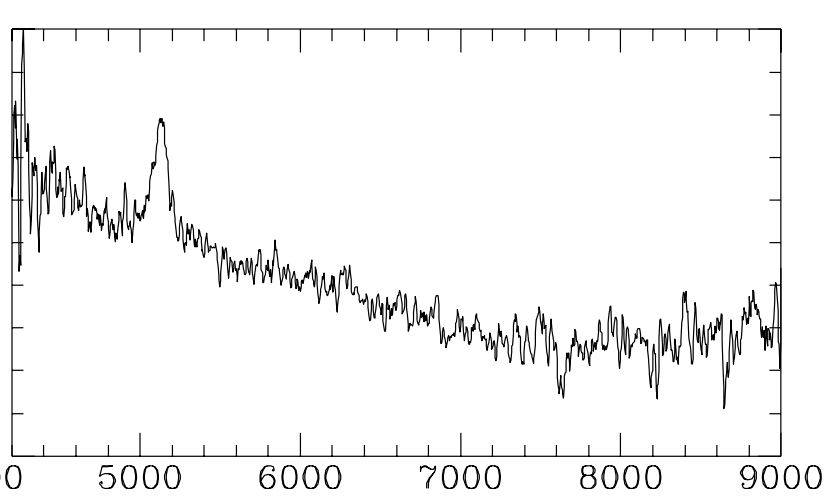

Wavelength $(\AA)$

Fig. 1. continued

the $0.5-2.0 \mathrm{keV}$ band), NVSS integrated flux density at $1.4 \mathrm{GHz}$ (in $\mathrm{mJy}$ ), observing set-up, date of the observation and total exposure time. The objects without the prefix "REX" in the name do not belong to the final REX catalog.

The X-ray flux has been derived from the count-rate using the value of Galactic $N_{\mathrm{H}}$ at the source position (Dickey \& Lockman 1990) and assuming $\alpha_{\mathrm{X}}=1$, which is the mean value expected for our sources. For some of the sources that are not in the final REX catalog, the $\mathrm{X}$-ray flux has been computed from the count-rate found in the 1RXP catalog (ROSAT NEWS No. 32, 1994), converted from the $0.1-2.4 \mathrm{keV}$ to $0.5-2.0 \mathrm{keV}$ band. These X-ray fluxes are indicated with an asterisk in Tables 2, 3 and 4 . The uncertainty on the X-ray fluxes is about $20 \%$. The typical rms of the radio (NVSS) maps is $0.45 \mathrm{mJy} /$ beam $^{1}$. We note that in the case of objects in cluster or in group of galaxies the X-ray flux is the sum of the active nucleus plus the extended thermal component.

\footnotetext{
${ }^{1}$ For a detailed description of the uncertainties associated to the radio flux densities of the NVSS catalog, see Condon et al. (1998).
} 


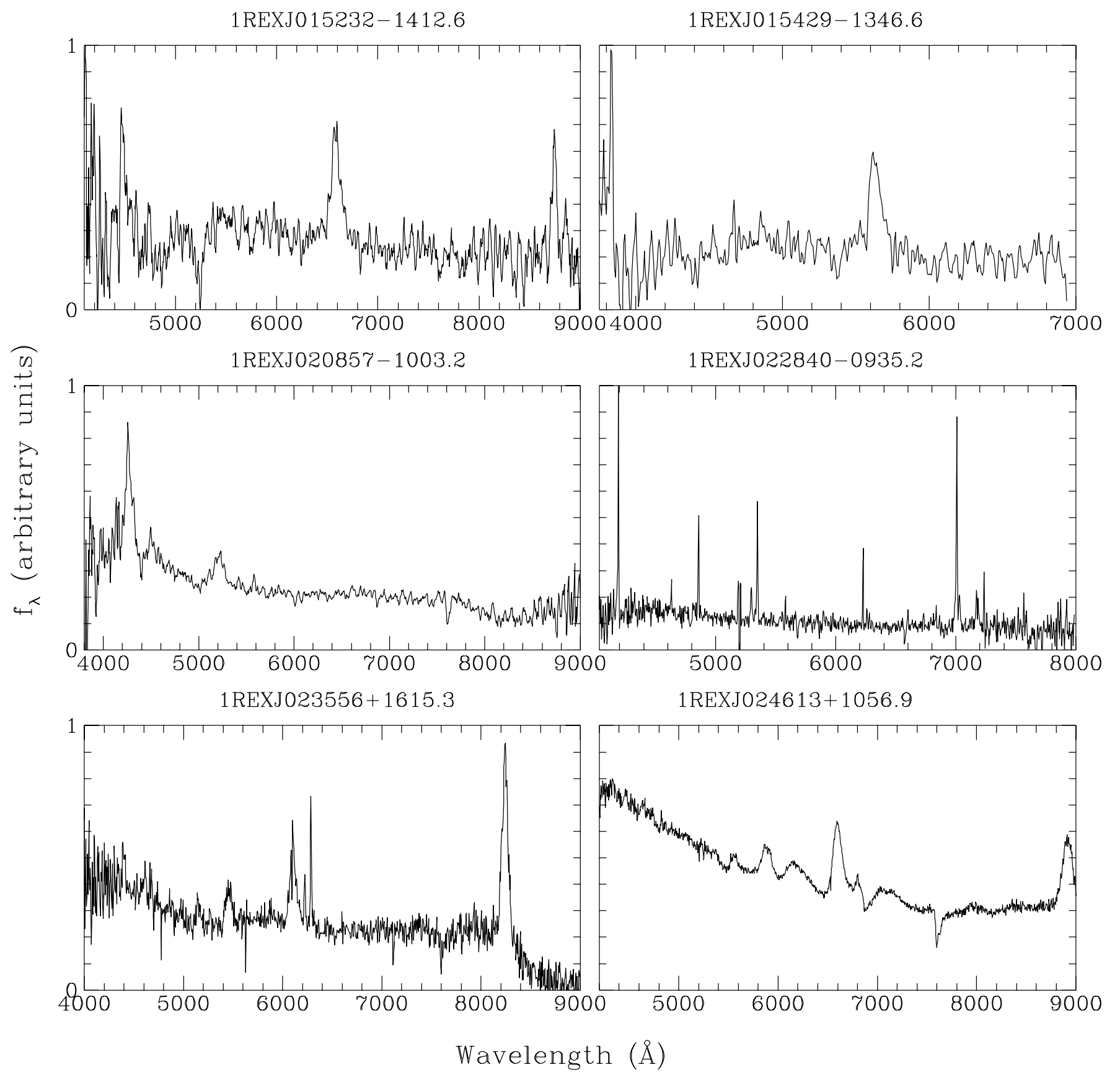

Fig. 1. continued

Given the available statistics and the spatial resolution of the PSPC, particularly poor in the external part of the field, in most cases we are not able to distinguish the two components. We have evidence, from the optical images, that 4 objects belong to a cluster/group of galaxies: we believe that in these cases the X-ray luminosity is mainly due to the diffuse intracluster gas (see Sect. 3).

\section{The Emission Line objects}

In this paper we present newly identified Emission Line objects. We define Emission Line objects those sources for which at least one strong $(\mathrm{EW} \gg 5 \AA$ in the source rest frame) emission line is present in the spectrum. This criterium excludes BL Lac objects and radiogalaxies without emission lines in their spectrum. There is evidence (Vermeulen et al. 1995; Corbett et al. 1996; Marchã et al. 1996) that some "true" BL Lacs might show emission lines with an equivalent width slightly larger than the "canonical" limit of $5 \AA$, proposed by Stocke et al. (1991). 
1REXJ030459+0002.5

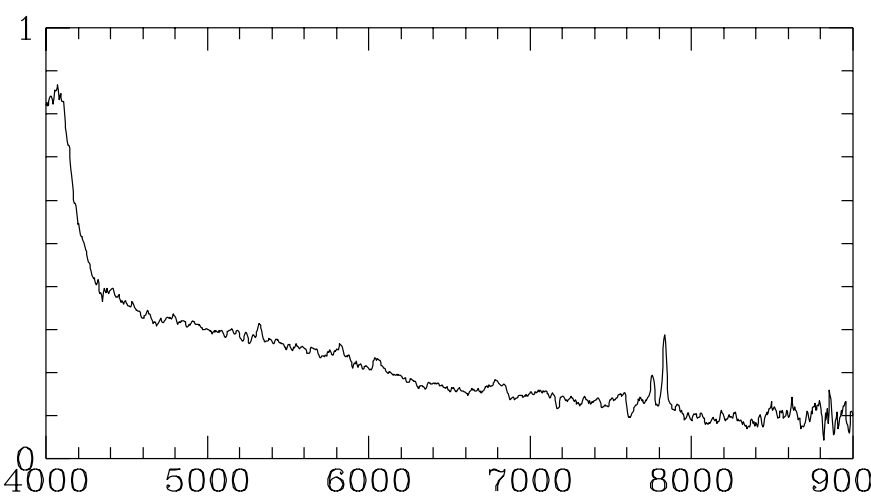

1REXJ034026-2234.9

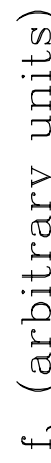

1REXJ031958+0355.9

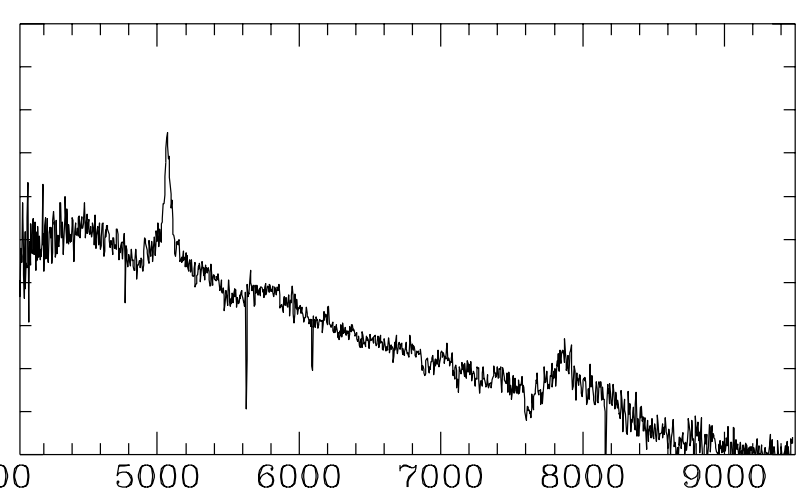

1REXJ035348-1020.2

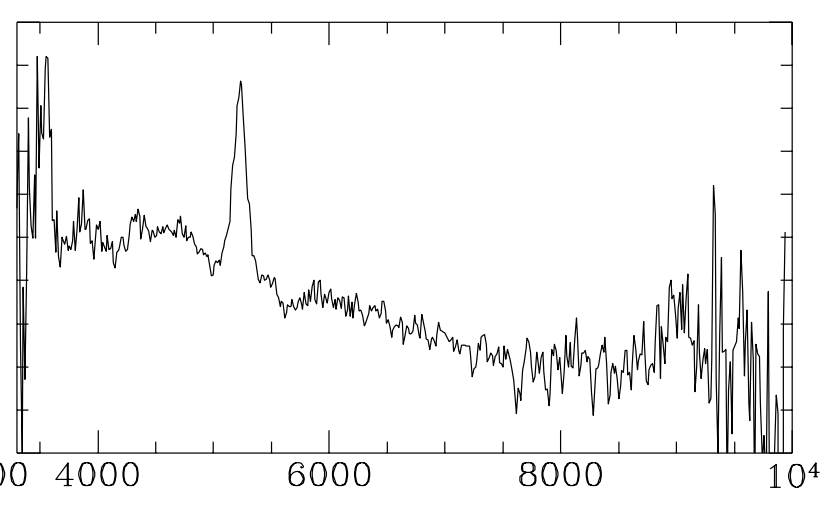

1REXJ041405-1224.2
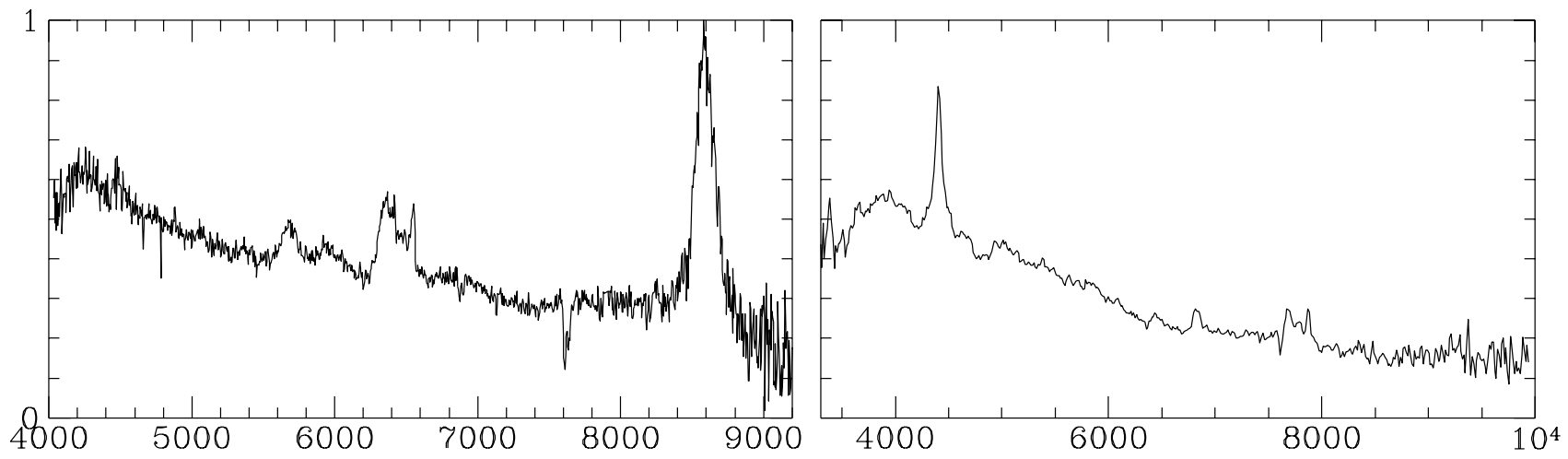

Wavelength $(\AA)$

Fig. 1. continued

However, the majority of the objects presented here show very strong emission lines well above the limit of $5 \AA$.

We further classify objects as Broad Emission Line (BEL) AGNs if at least one line has a $F W H M>1000 \mathrm{~km} \mathrm{~s}^{-1}$ (in the source rest frame), or Narrow Emission Line (NEL) object if all the observed lines have $F W H M<1000 \mathrm{~km} \mathrm{~s}^{-1}$.

This classification reflects the common division between "type 1" (i.e. Broad Line) and "type 2" (Narrow Line) AGNs often discussed in literature. The majority of the EL objects presented here is "type 1" AGNs.
In one case (1REXJ213248-0219.8), the FWHM of $\mathrm{H} \beta$ and $\mathrm{H} \alpha$ is $\sim 1300-1400 \mathrm{~km} \mathrm{~s}^{-1}$, thus slightly higher than the limit used to classify the object as NEL AGN. This value of FWHM and the ratio between [OIII] and $\mathrm{H} \beta \quad(=1.4)$ suggests a classification as narrow-line Seyfert 1 galaxy (NLS1, [OIII]/H $\beta<3$ and $F W H M<2000 \mathrm{~km} \mathrm{~s}^{-1}$, e.g. Osterbrock \& Pogge 1985). In the few cases of Narrow Line objects, we have applied, when possible, the diagnostic criteria described in Veilleux \& Osterbrock (1987) to separate a starburst galaxy from an AGN or LINER. However it is worth 


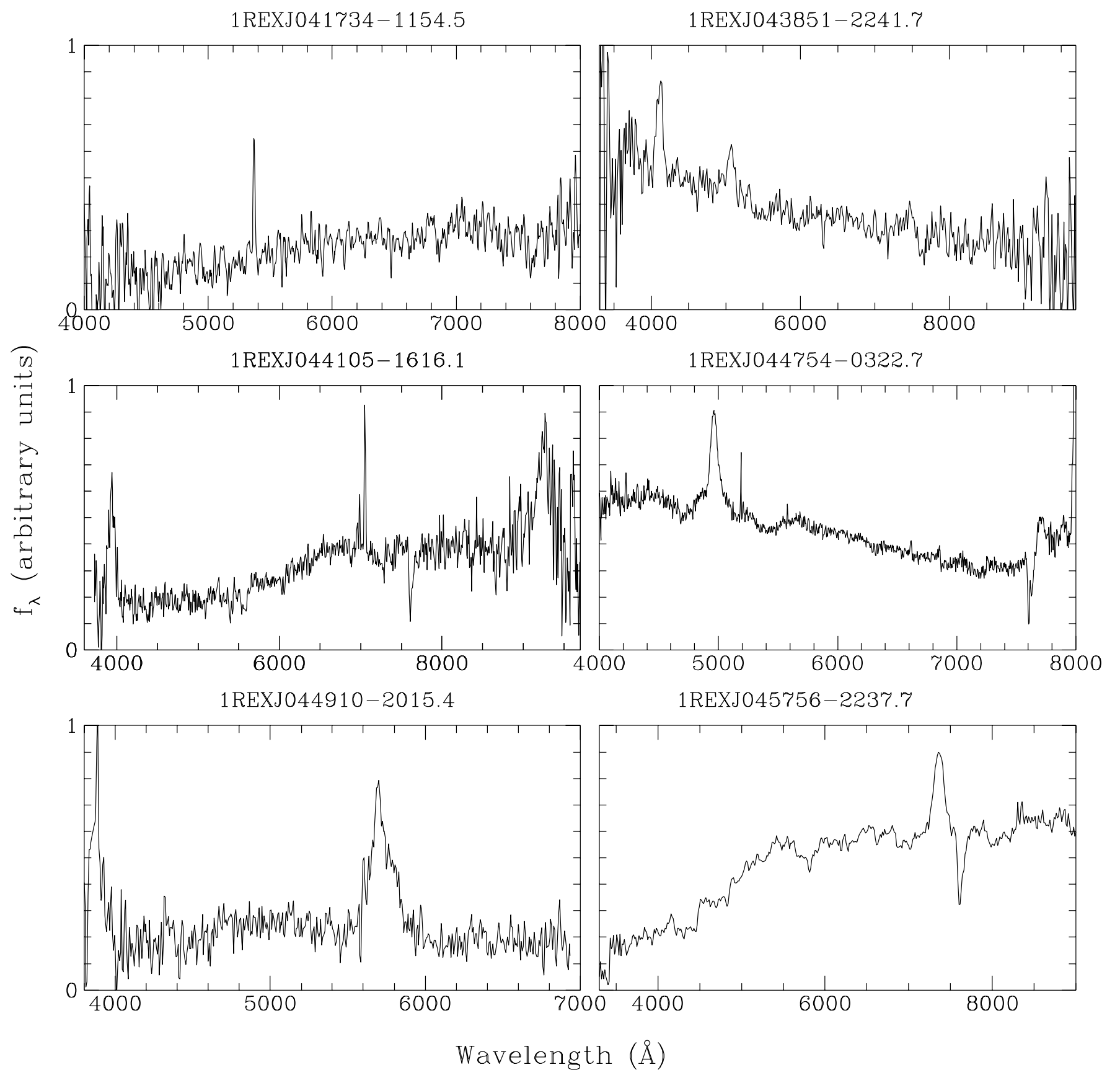

Fig. 1. continued

noting that, as discussed in Veilleux \& Osterbrock (1987), the separation between starburst galaxies and AGNs is not very sharp and a mixture of both phenomena could be present in the same object (Hill et al. 1999; Goncalves et al. 1998). Given the quality of our spectra, we didn't attempt to estimate a correction for reddening. However, for the classification of these objects we have used, as long as possible, the ratios between couples of emission lines like $[\mathrm{OIII}] \lambda 5007 / \mathrm{H} \beta,[\mathrm{NII}] \lambda 6583 / \mathrm{H} \alpha$, $[\mathrm{SII}] \lambda 6716+\lambda 6731 / \mathrm{H} \alpha$ that are not very reddening sensitive.
We briefly discuss here the 4 NEL objects for which we can apply the Veilleux \& Osterbrock (1987) diagnostic criteria.

1REXJ005924+2703.5: the values of the ratios between $[\mathrm{SII}] \lambda 6716+\lambda 6731$ and $\mathrm{H} \alpha(\mathrm{Log}([\mathrm{SII}] / \mathrm{H} \alpha)=-0.29)$ and between $[\mathrm{OIII}] \lambda 5007$ and $\mathrm{H} \beta(\log ([\mathrm{OIII}] / \mathrm{H} \beta)=0.02)$ put this object in the zone intermediate between AGNs and HII regions.

1REXJ022840-0935.2: the intensity ratio between $[\mathrm{OIII}] \lambda 5007$ and $\mathrm{H} \beta(\log ([\mathrm{OIII}] / \mathrm{H} \beta)=0.26)$ and between $[\mathrm{NII}] \lambda 6583$ and $\mathrm{H} \alpha(\log ([\mathrm{NII}] / \mathrm{H} \alpha)=-0.9)$ suggests a 
1REXJ053628-3401.1

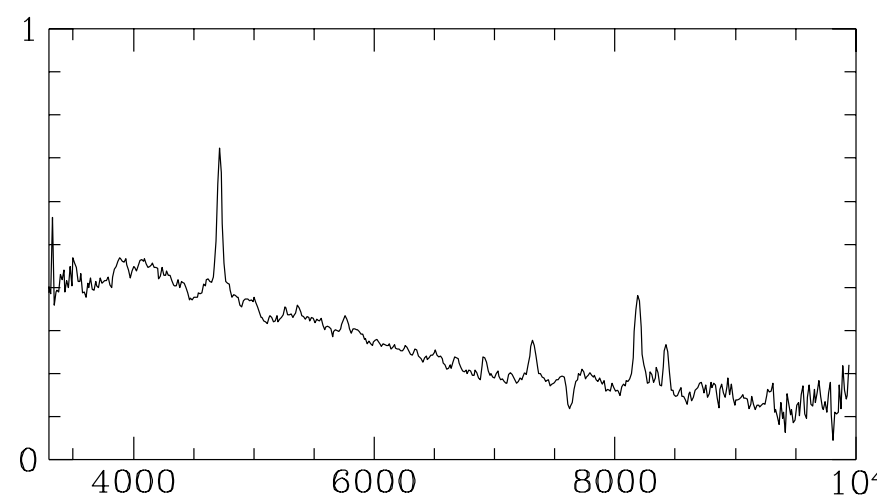

1REXJ055722-1414.7
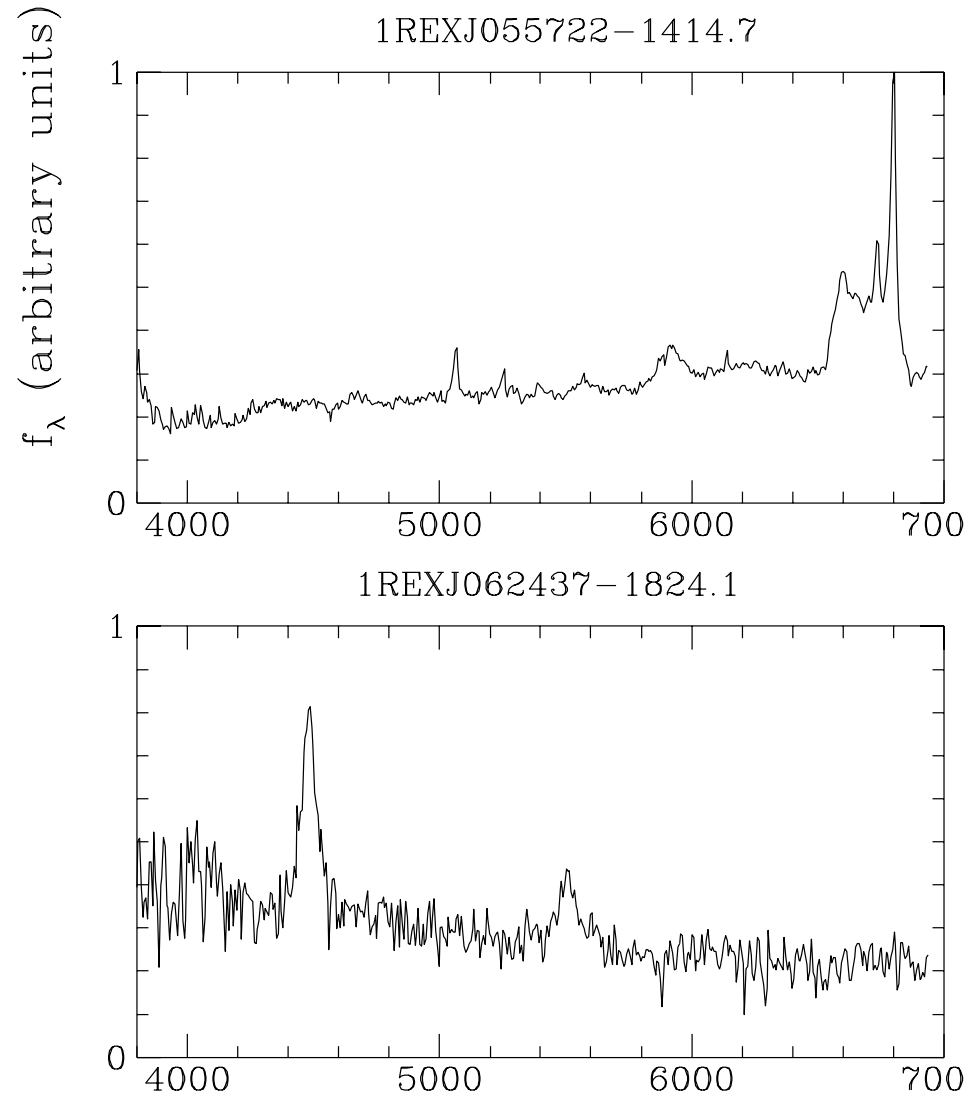

1REXJ054129-3427.7

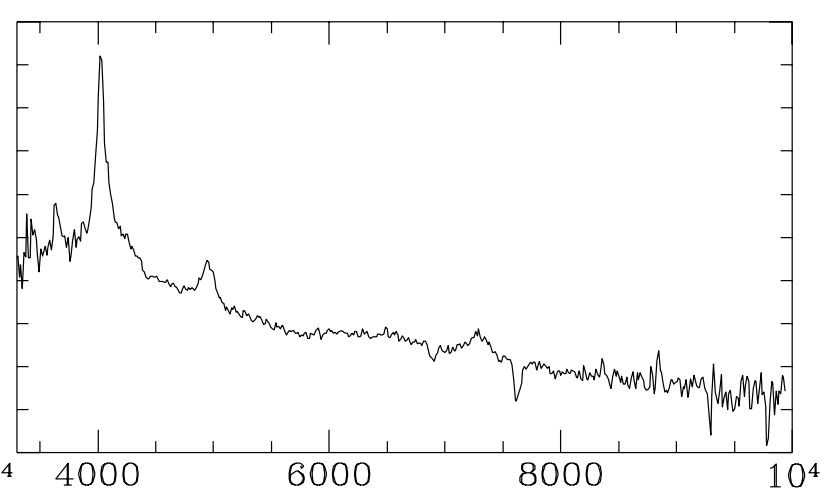

1REXJ061757+7816.1

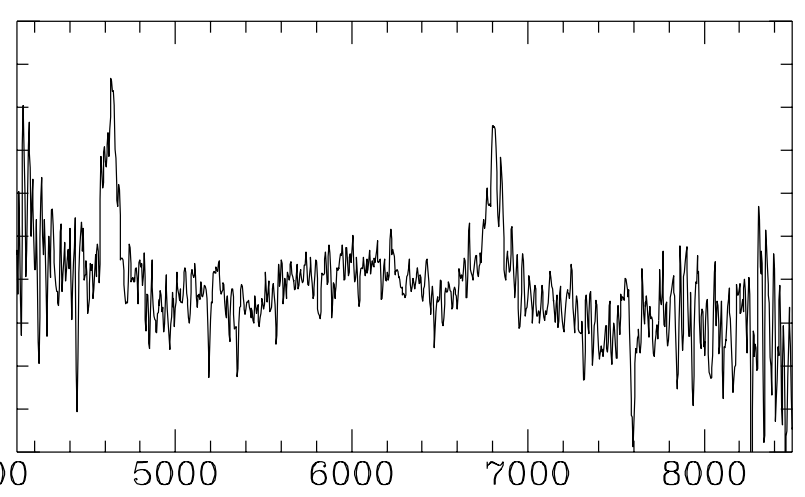

1REXJ065154+6955.4

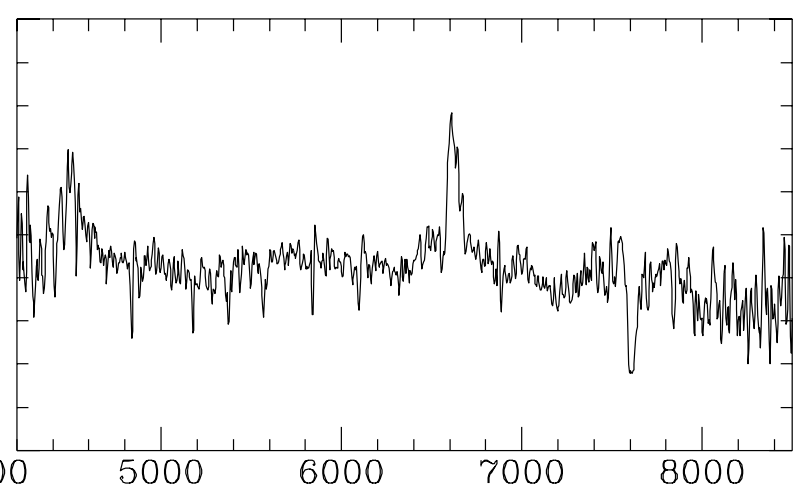

Wavelength $(\AA)$

Fig. 1. continued

classification as H II region or starburst galaxy rather than AGN for this object.

1REXJ121815+0744.4: the $\mathrm{H} \alpha$ is blended with [NII] $\lambda 6548$ and it is difficult to measure its width. The $F W H M$ is about $800-1000 \mathrm{~km} \mathrm{~s}^{-1}$. The strong intensity of the $[\mathrm{OIII}] \lambda 5007$ line if compared with the $\mathrm{H} \beta(\log ([\mathrm{OIII}] / \mathrm{H} \beta)=0.88)$ and the ratio between $[\mathrm{SII}] \lambda 6716+\lambda 6731$ and $\mathrm{H} \alpha(\log ([\mathrm{SII}] / \mathrm{H} \alpha)=-0.37)$ strongly suggest the presence of an AGN.

1REXJ230311-0859.3: the $\mathrm{H} \alpha$ is blended with [NII] $\lambda 6583$. The FWHM of the $\mathrm{H} \alpha$ is about $1000 \mathrm{~km} \mathrm{~s}^{-1}$ although a proper de-blending is necessary for a more accurate measurement. The measured value of $\log ([\mathrm{N}$ $\mathrm{II}] \lambda 6583 / \mathrm{H} \alpha) \sim-0.15$ and the lack of $[\mathrm{SII}] \lambda 6716+\lambda 6731$ lines lead to a tentative classification of this source as Narrow Emission Line galaxy (NELG). This object belongs to a galaxy pair.

For the remaining 4 NEL objects (1REXJ0417341154.5, 1REXJ220451-1815.5, 1REXJ235139-2605.0, J014716-0008.2) we cannot apply the diagnostic criteria described above. These objects typically show [OII] $\lambda 3727$ and $[\mathrm{OIII}] \lambda 5007$ lines. All these 4 NEL objects show 
1REXJ071859+7124.3

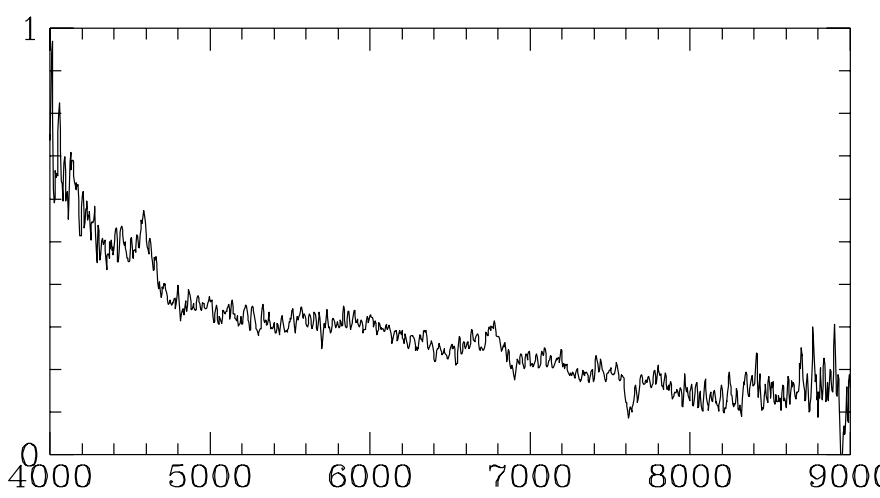

1REXJ081041+0810.0

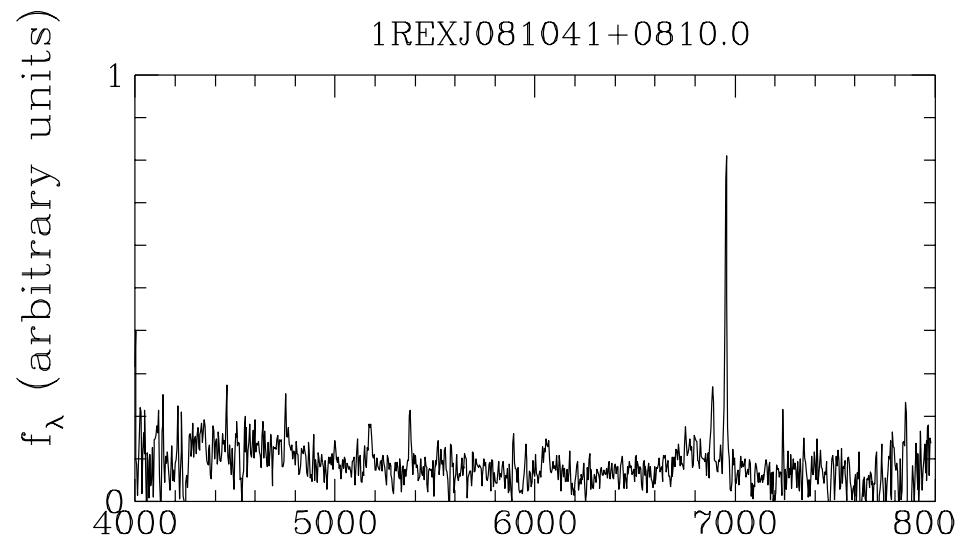

1REXJ082656+6542.5

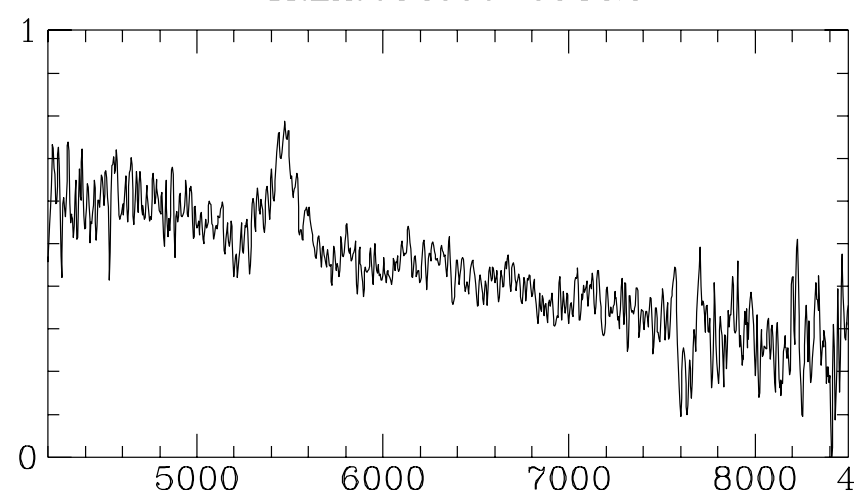

Wavelength
1REXJ073125+6718.7

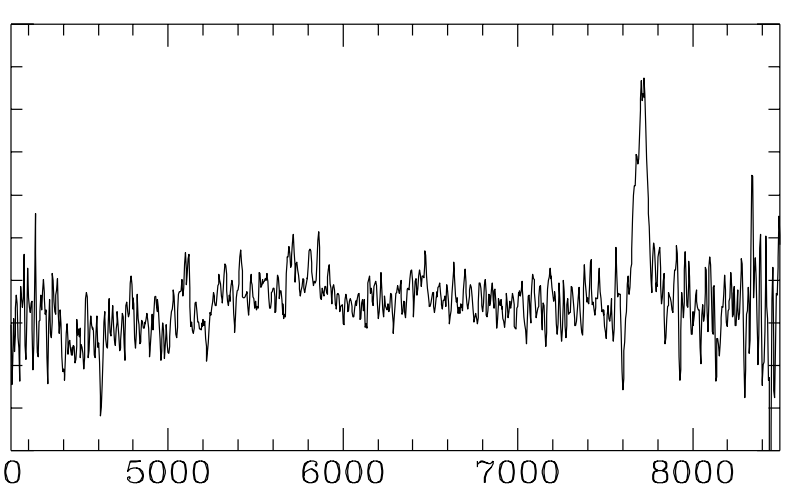

1REXJ081108+4533.8

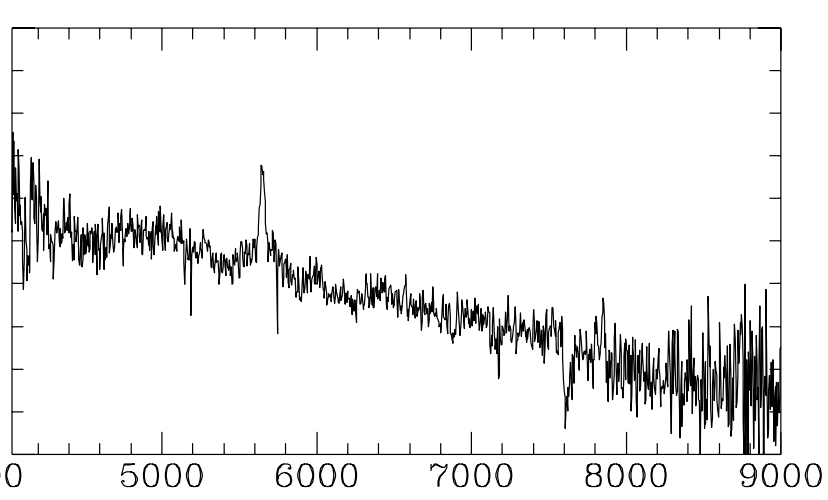

1REXJ082733+2637.2

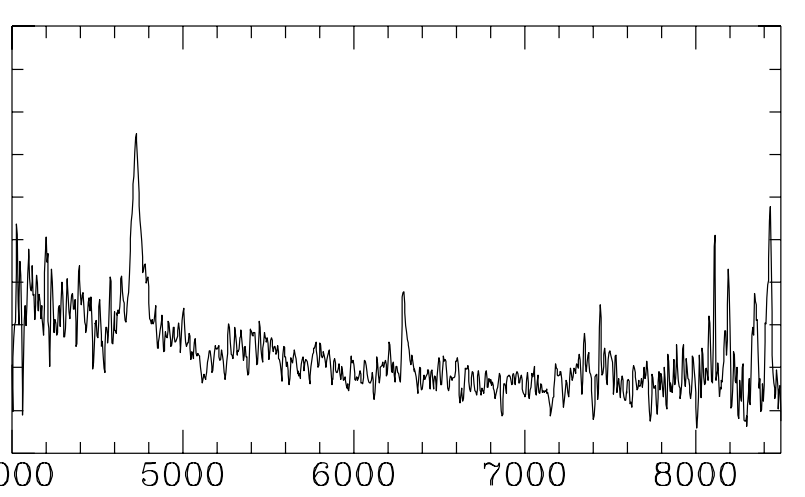

$(\AA)$

Fig. 1. continued

an X-ray luminosity exceptionally high for this class of source $\left(L_{\mathrm{X}}>10^{44} \mathrm{erg} \mathrm{s}^{-1}\right)$. One of these sources, 1REXJ235139-2605.0, is the cD galaxy of the cluster A 2667 which is very luminous in the X-ray band $\left(2.110^{45}\right.$ $\mathrm{erg} \mathrm{s}^{-1}$ in the $0.1-2.4 \mathrm{keV}$ band, Rizza et al. 1998). In other two cases (1REXJ041734-1154.5, 1REXJ2204511815.5) the CCD image taken at the UH $88^{\prime \prime}$ telescope reveals the presence of an overdensity of galaxies around $\left(<1^{\prime}\right)$ the radio source thus suggesting that these objects belong to a cluster/group of galaxies. Finally, J014716-0008.2 is probably in a cluster since at least two companions at the same redshift of the radio source, have been found. In conclusion, all the NEL objects presented in this paper with an X-ray luminosity greater than $10^{44} \mathrm{erg} \mathrm{s}^{-1}$ probably belong to a cluster/group of galaxies. In these cases, the very high $\mathrm{X}$-ray luminosity is probably originated from the intracluster gas and not from the galaxy itself. Further X-ray observations with higher spatial resolution are required in order to measure the true luminosity of the object. On the contrary, all the isolated NEL objects have a low luminosity in the 
1REXJ085120+1358.3

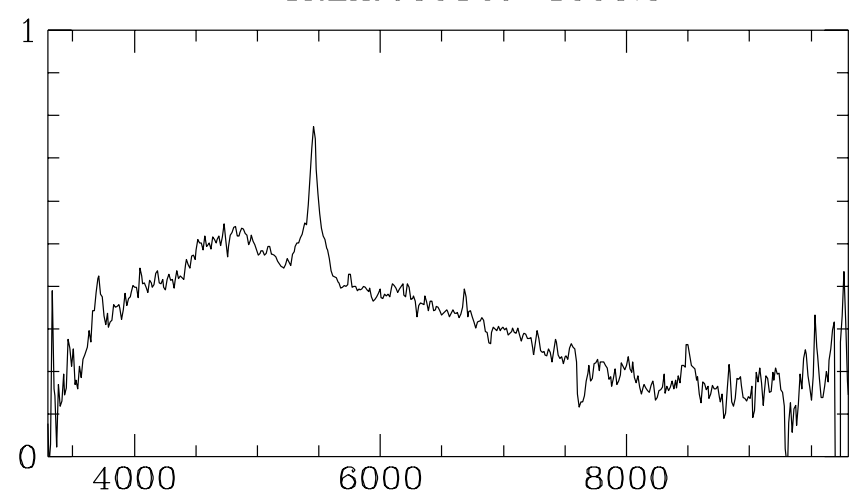

1REXJ085312+1358.8

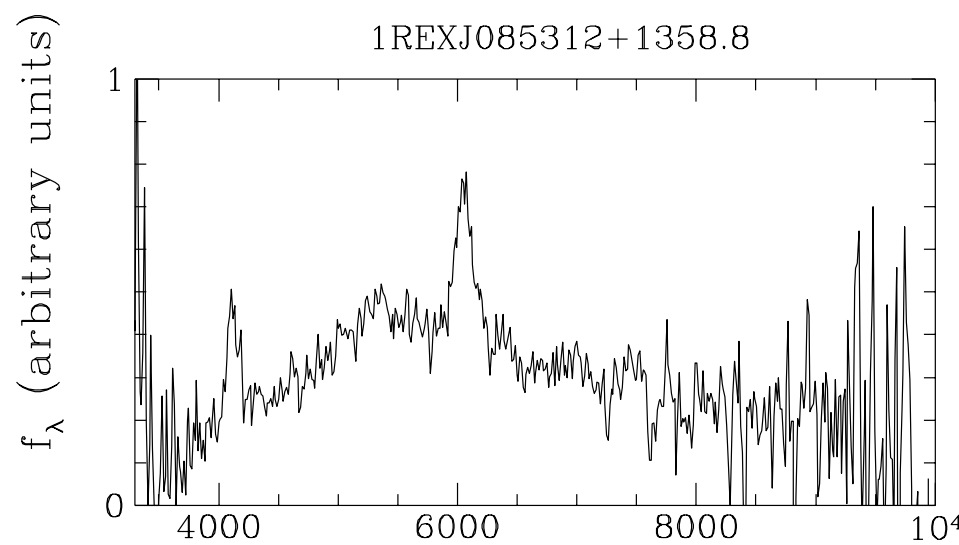

1REXJ092655-2345.4

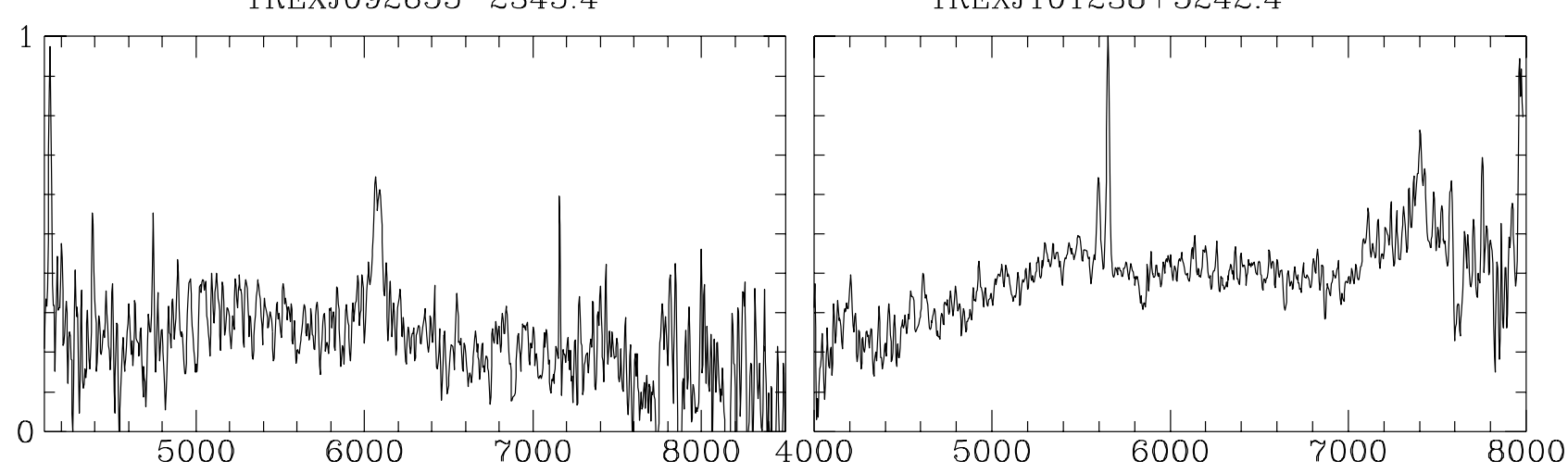

1REXJ085211+7627.3

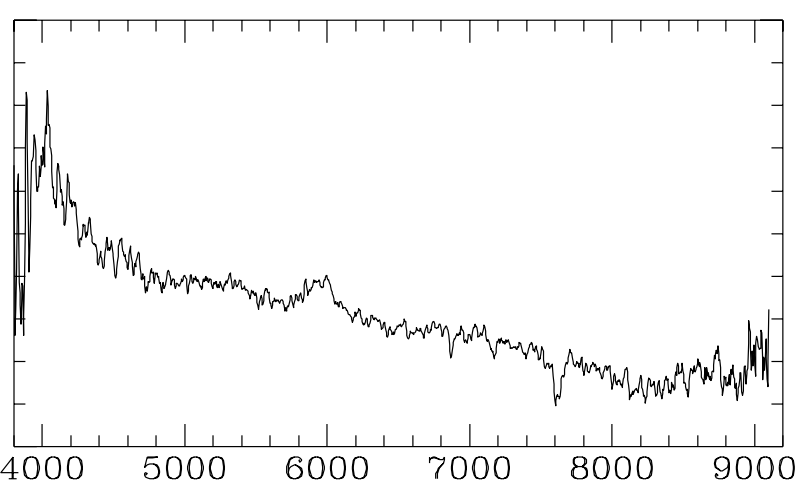

1REXJ090015-2817.9

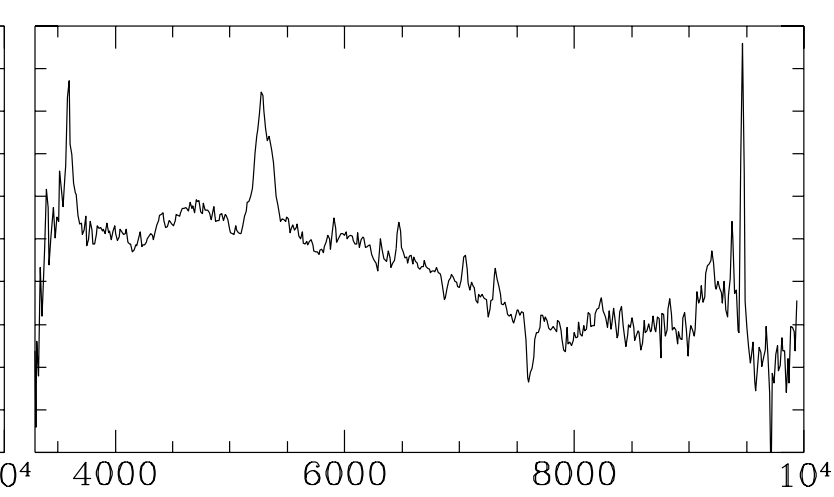

Wavelength $(\AA)$

Fig. 1. continued

radio and in the X-ray band $\left(L_{\mathrm{X}}<210^{43} \mathrm{erg} \mathrm{s}^{-1}\right.$, $\left.P_{1.4} \leq 10^{31} \mathrm{erg} \mathrm{s}^{-1} \mathrm{~Hz}^{-1}\right)$.

The classification of the EL objects in Broad Line (B) and Narrow Line (N) objects is reported in Table 5 (Col. 2) together with the measured redshift and the relative confidence (Cols. 3 and 4), X-ray and radio luminosities (Cols. 5 and 6), the observed features in emission (Col. 7) and in absorption (Col. 8). The luminosities have been computed and K-corrected under the assumption of power-law spectra $\left(f_{\nu} \propto \nu^{-\alpha}\right)$. In the radio band we have used the spectral index $\left(\alpha_{R}\right)$ as computed between $1.4 \mathrm{GHz}$ (NVSS) and $5 \mathrm{GHz}$ if the source is included in the GB6 (Gregory \& Condon 1991) or in the PMN (Griffith \& Wright 1993) catalog. For three sources (1REXJ061757+7816.1, 1REXJ082656+6542.5, 1REXJ085211+7627.3) not present in the GB6 and PMN catalogs, we have used the flux at $365 \mathrm{MHz}$ derived from the WENSS survey (Rengelink et al. 1997) to estimate the radio spectral index. Finally, if no fluxes at frequencies different from $1.4 \mathrm{GHz}$ are available, we have used the lower limit on $\alpha$ computed taking into account the proper flux density limit at $5 \mathrm{GHz}$ for the 


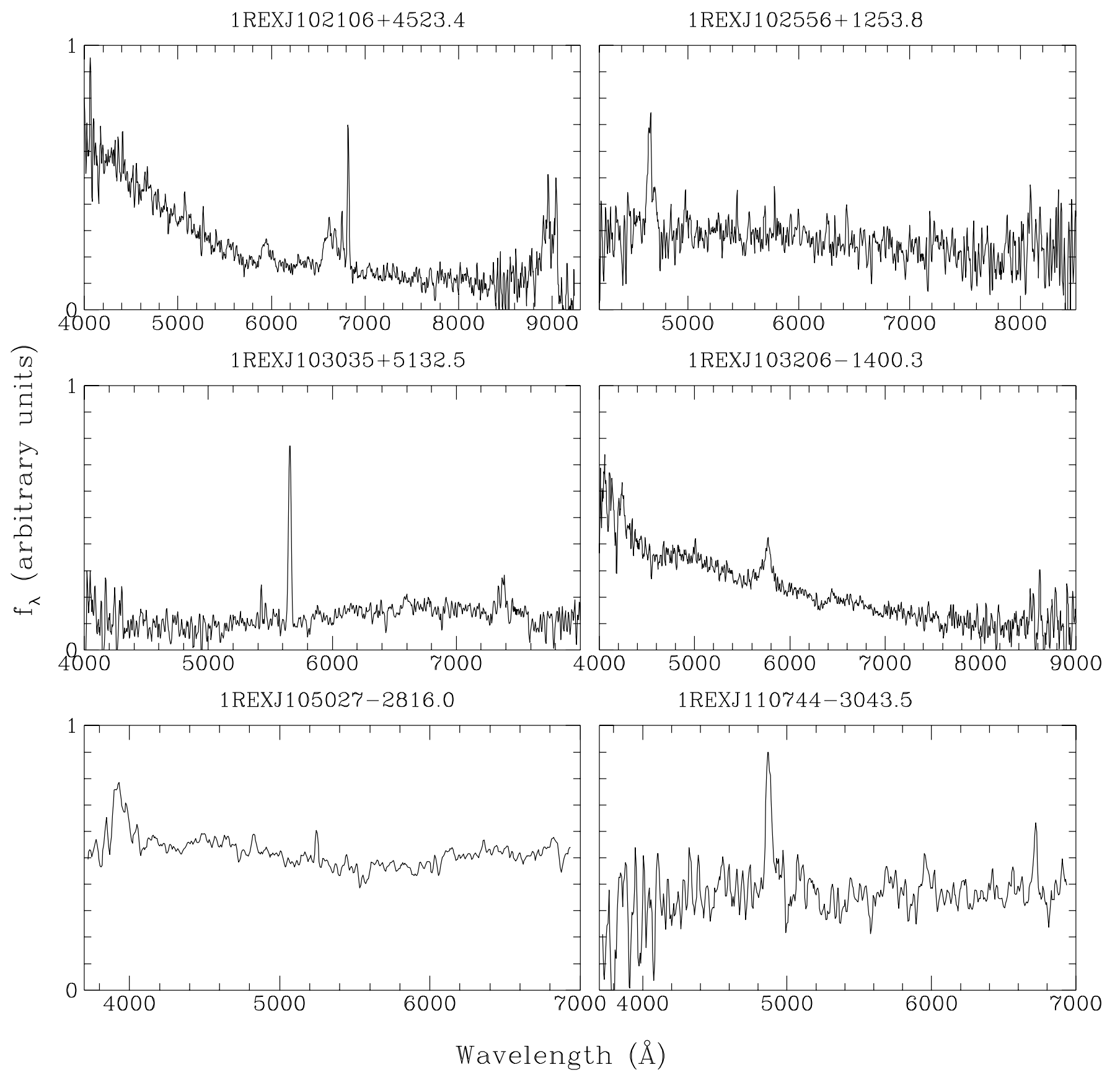

Fig. 1. continued

GB6 ( $18 \mathrm{mJy}$ ) and PMN catalogs (from 40 to $72 \mathrm{mJy}$ depending on the declination). These monochromatic luminosities (indicated by an asterisk in Table 5) should be considered as lower limits. The actual value of the luminosity is close to these values for low-redshift objects $(z<0.5)$ while it can change up to a factor of 10 for those sources at $z>1$.

In the X-ray band, the assumption of $\alpha_{\mathrm{X}}=1$ is adequate to convert the count-rate into an X-ray flux (error less than 20\%) but it is not good enough for the
K-correction, in particular for high redshift objects. Thus, we have used the relationships found in Brinkmann et al. (1997) that give the $\alpha_{\mathrm{X}}$ as a function of the redshift for a sample of X-ray selected radio-loud AGNs found in the ROSAT All Sky Survey (RASS). In particular, we have used the appropriate relationship for steep spectrum (SS, $\left.\alpha_{\mathrm{R}}>0.5\right)$ and flat spectrum (FS, $\left.\alpha_{\mathrm{R}}<0.5\right)$ radio sources given in Brinkmann et al. (1997) on the basis of the radio slope between $1.4 \mathrm{GHz}$ and $5 \mathrm{GHz}$. If a radio slope is not available, we have used the relationship between $\alpha_{\mathrm{X}}$ and $z$ proper for the SS, if the lower limit on $\alpha_{\mathrm{R}}$ (based on the 


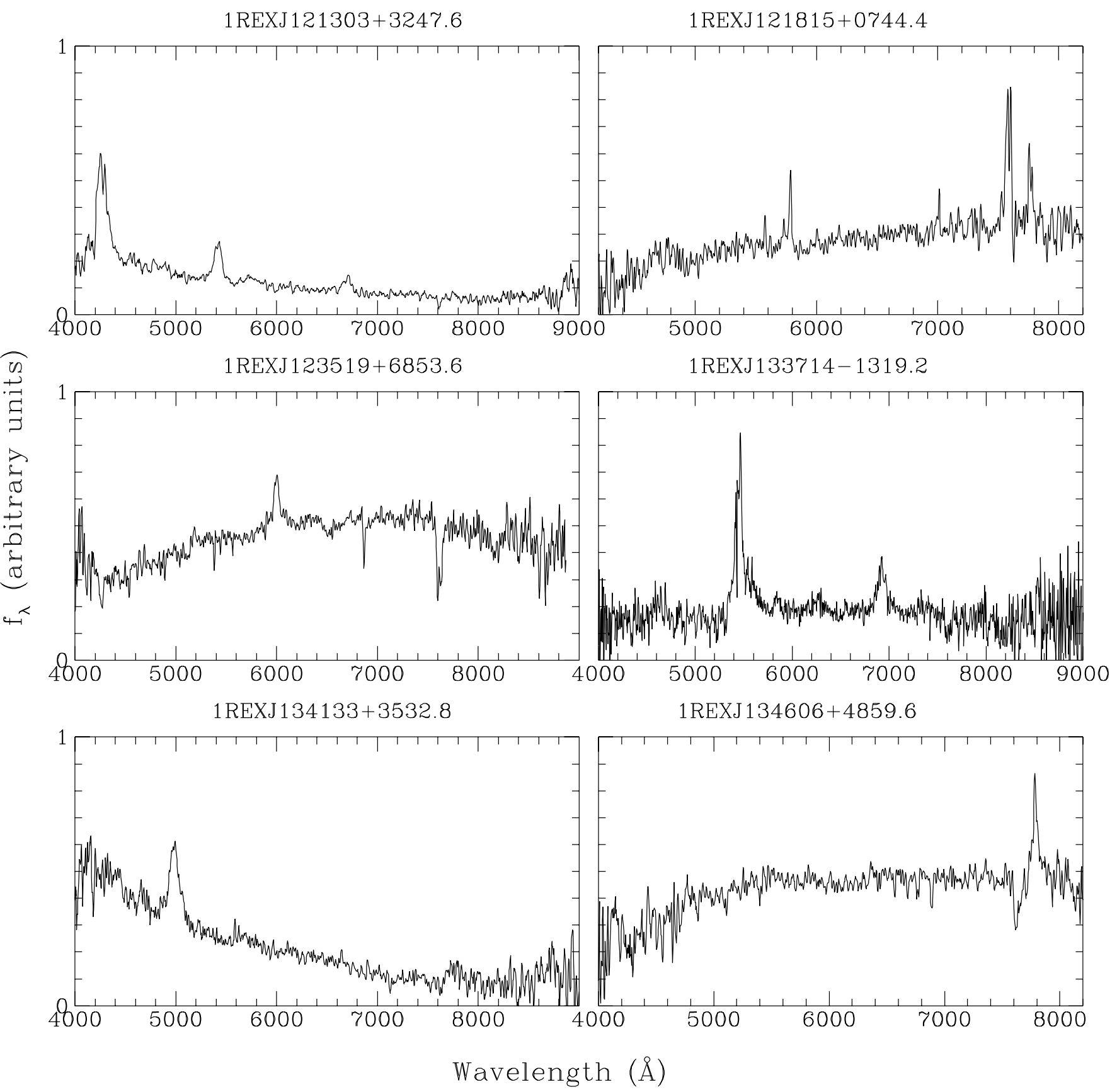

Fig. 1. continued

GB6 or PMN flux limits) is larger than 0.5; otherwise, we have used the formula for the FS. The used values of $\alpha_{\mathrm{X}}$ range from 0.8 to 1.15 .

A separate table (Table 6) contains the EL objects that are not included in the final version of the REX catalog. These sources have been excluded because they do not satisfy anymore the final selection criteria of the REX survey described in Caccianiga et al. (1999).

We note that the spectra presented here have been collected with the primary aim of identifying the sources and measuring the redshifts. The nominal error on the redshift is $\sim 0.001$ (at $5000 \AA$ ) but in many cases, given the signalto-noise ratio of the spectrum, the error is larger (about $0.005-0.01)$. When only one strong emission line is present in the spectrum, we have assumed, as usual, that this feature is the MgII $\lambda 2798$. We have flagged as tentative the values of redshift computed in these cases (a letter "T" after the value of redshift in Tables 5 and 6 ). We have also flagged as tentative the redshift of 1REXJ220451-1815.5 for which we have assumed that the two observed features are $[\mathrm{OII}] \lambda 3737$ and $[\mathrm{OIII}] \lambda 5007$ respectively. 


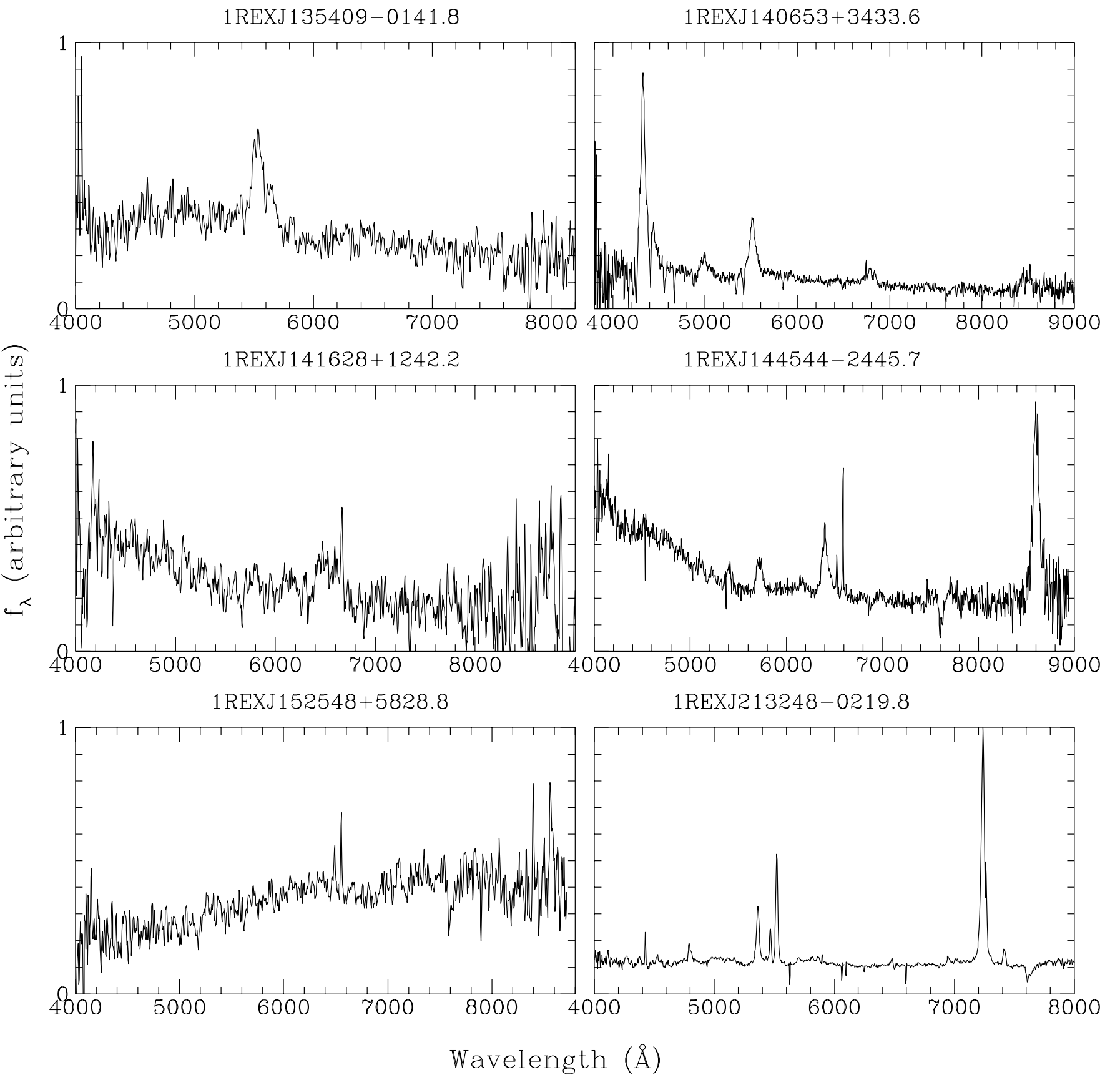

Fig. 1. continued

All the sources presented here were unidentified when we observed them. During the preparation of this paper, 12 sources have been observed and identified independently by other authors, in particular during the identification of other surveys that combine X-ray and radio data (i.e. the DXRBS survey, Perlman et al. 1998 and the RGB survey, Laurent-Muehleisen et al. 1998). Moreover, in two additional cases we have intentionally re-observed the object because the redshift was based on poor quality data (i.e. low dispersion prism observations, 1REXJ005229-3743.8) or we wanted to assess the presence of the $\mathrm{H}_{\alpha}$ in emission (1REXJ230311-0859.3). Except for 1REXJ005229-3743.8, for which we have found a redshift significantly different from the one published (1.60 instead of 2.25), in all the other cases we confirm the published values of redshift, with discrepancies below $3 \%$ and only in one case of $6 \%$. A footnote in the source name in Tables 5 and 6 indicates that an optical identification and a redshift for that object have been already published in literature.

In Figs. 1 and 2 we present the spectra of all the EL objects included (Fig. 1) and not included (Fig. 2) in the REX catalog. The $y$ axis (the flux) has arbitrary units. 
1REXJ220451-1815.5

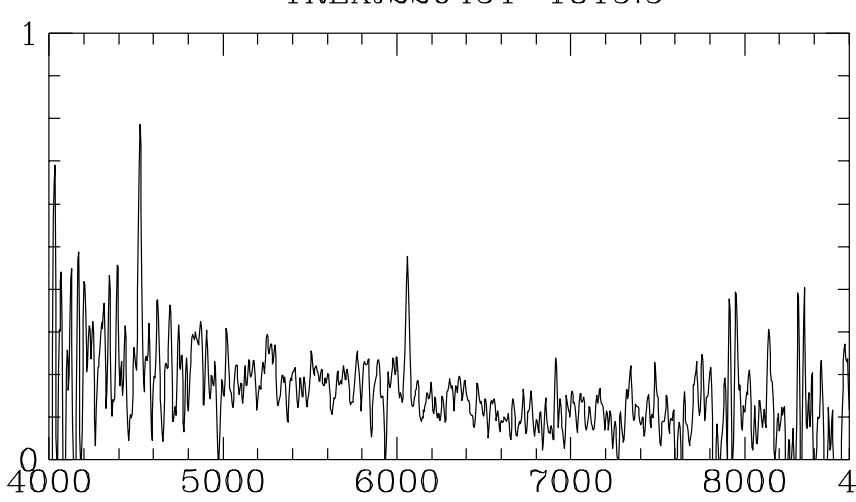

1REXJ23031 1-0859.3

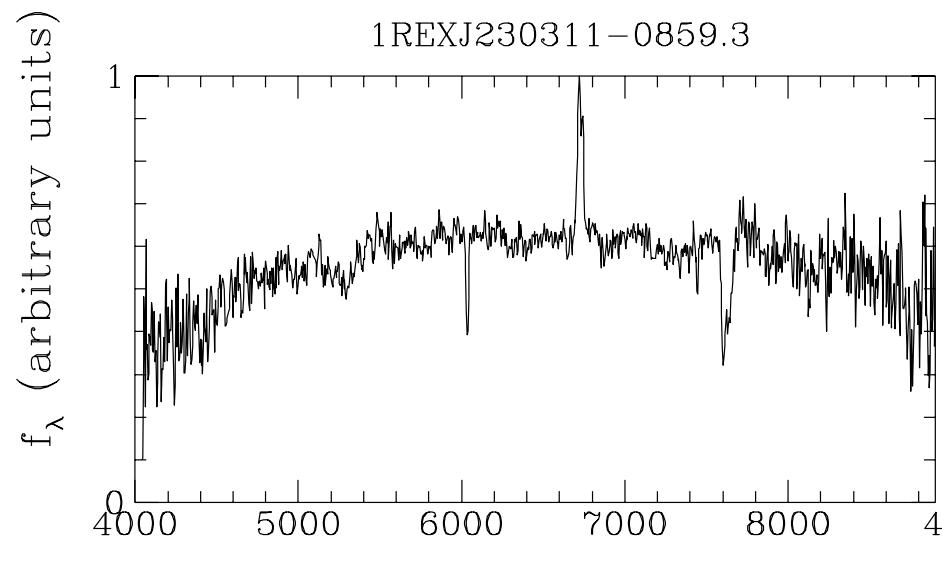

1REXJ235139-2605.0

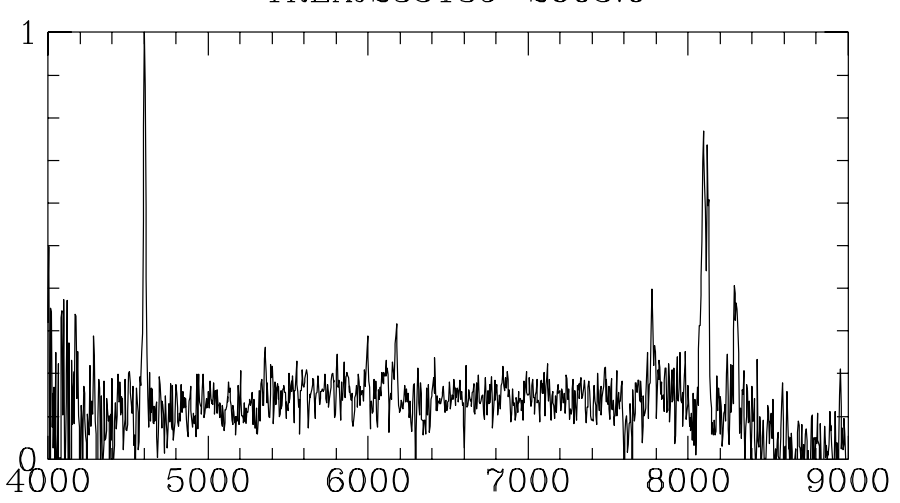

Wavelength $(\AA)$
1REXJ223313+3405.0

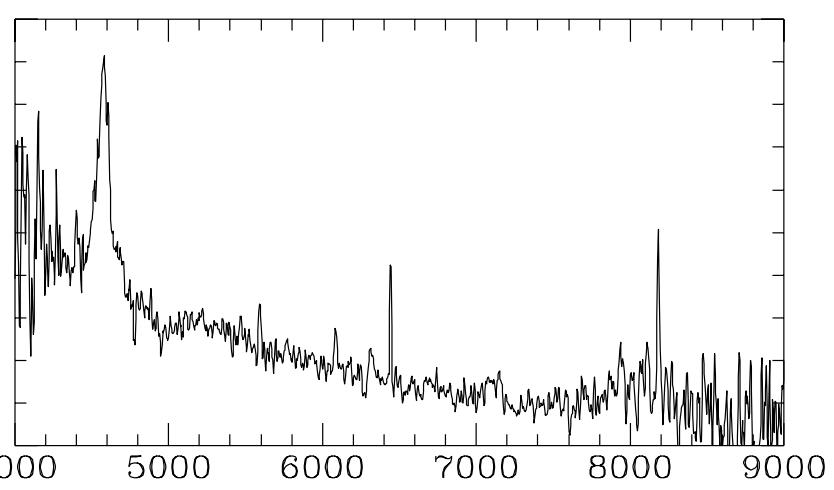

1REXJ235029-2620.7

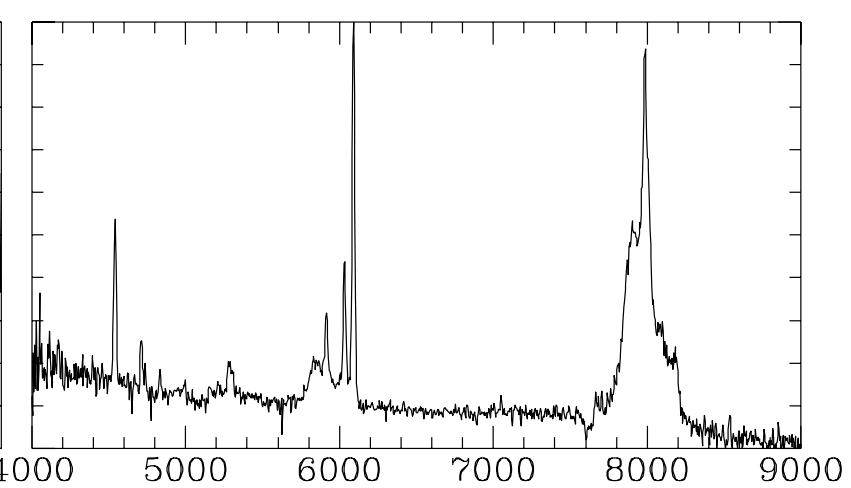

Fig. 1. continued

\section{Discussion}

Although the objects presented here do not represent a complete sample, since the optical identification of the REX survey is still in progress, it is interesting to investigate what kind of AGNs is selected by combining an X-ray and a radio survey. In order to increase the statistics, we have used for this analysis all the Emission Line AGNs discovered in the REX survey so far. In particular, from the 232 EL objects available, we have selected the 226 with a firm determination of redshift. The majority of the objects identified from literature is composed by QSOs, i.e. type 1 objects. For the low-redshift objects the situation is less clear in particular if we want to apply uniformly the same criteria used for the sources observed by us. For this reason, in the following analysis we will not distinguish between Narrow and Broad Emission Line objects.

For all these sources we have computed the ratio $(r)$ of the integrated to the peak NVSS flux densities, resulting from the Gaussian fit to the image (see Condon et al. 1998 for details); $r$ is a good indicator of the compactness of the source at the survey resolution. The majority (74\%) of the sources has a $r$ below 1.1, i.e. they are unresolved 


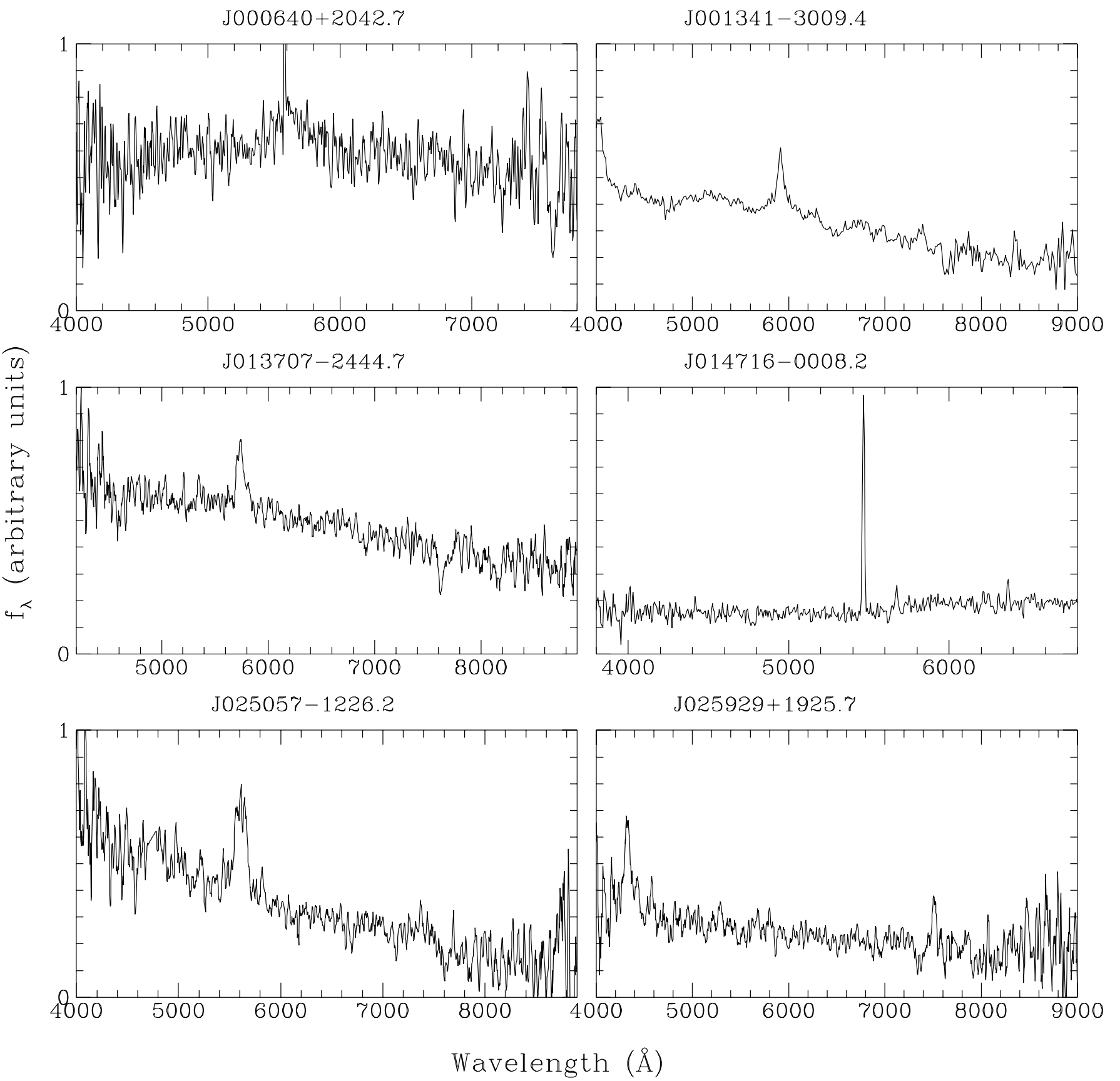

Fig. 2. Optical spectra of the 19 EL AGN not included in the REX survey

in the NVSS maps. We note, however, that the NVSS survey has been carried out with the VLA in the less resolved configuration (DnC) and, thus, the corresponding beam is quite large $\left(F W H M=45^{\prime \prime}\right)$. This beam, at redshift of 0.9 (which is the mean $z$ value of the 226 sources) corresponds to a linear size of about $470 \mathrm{kpc}$. Thus, even a typical lobedominated source at this redshift could be unresolved in the NVSS.

In Fig. 3 we have reported the histogram of the spectral indices of the 151 sources detected at $5 \mathrm{GHz}$ in the GB6 or PMN catalog. Ninety-four objects (62\%) are FS radio sources $(\alpha \leq 0.5)$ and $57(38 \%)$ are SS radio sources. Since compact, flat spectrum radio sources have a higher probability of being detected at $5 \mathrm{GHz}$ (in the GB6 or PMN catalogs) we expect that our distribution is biased against steep spectrum sources. For this reason we have considered the 59 sources with a flux density at $1.4 \mathrm{GHz}$ greater than $200 \mathrm{mJy}$ and falling in the area of sky covered by the GB6 catalog (which is deeper than PMN): all these sources should be detectable at $5 \mathrm{GHz}$ even if their radio spectrum is very steep $\left(\alpha_{\mathrm{R}} \sim 2\right)$. These sources, represented by the shaded area in Fig. 3, are equally 


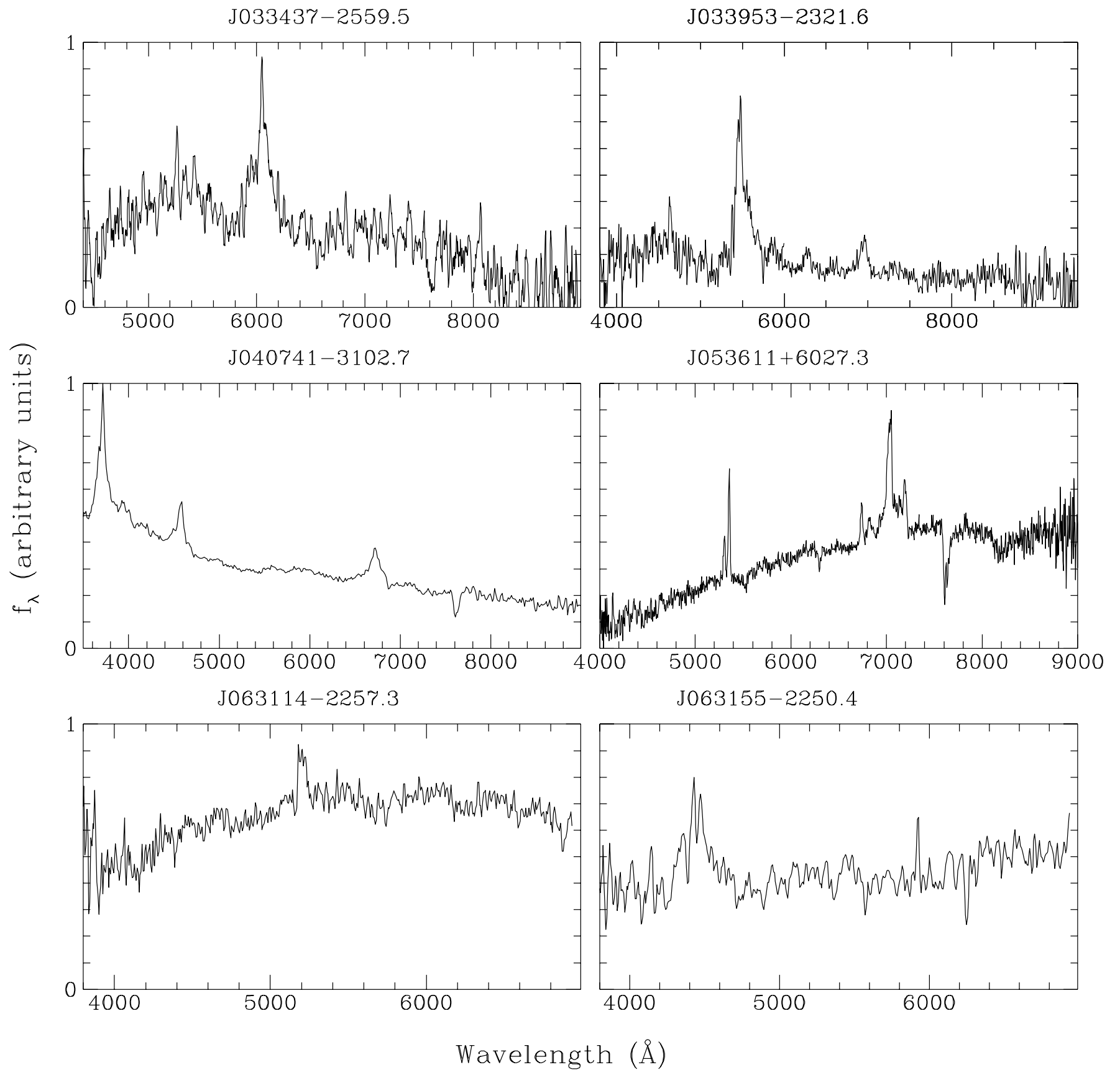

Fig. 2. continued

distributed between FS (51\%) and SS (49\%) radio sources. Their average radio slope is 0.41 .

The redshift distribution of the 226 AGN is presented in Fig. 4. The majority $(96 \%)$ of the sources have a redshift below 2 . The mean value of $z(=0.9)$ is the same found for the RL AGN in the EMSS (Della Ceca et al. 1994).

In Fig. 5 we present the monochromatic radio luminosities at $1.4 \mathrm{GHz}$ versus the X-ray luminosities in the $0.5-2.0 \mathrm{keV}$ band. We have considered here only the 151 AGNs for which we have computed the radio spectral index in order to reduce the uncertainties on the determination of the K-corrected radio luminosity which could be large for high redshift objects. Figure 5 shows a rather strong correlation between the radio and the X-ray luminosities. A least squares fit to the data gives $P_{1.4} \propto$ $L_{\mathrm{X}}^{1.06 \pm 0.04}$ (solid line in Fig. 5). This result is consistent (within $2 \sigma$ ) with a linear $\left(P_{1.4} \propto L_{\mathrm{X}}\right)$ correlation between the two luminosities. The Spearman Rank-Order correlation coefficient gives a highly significant probability $(>99.9 \%)$ that the two luminosities are correlated. However, a spurious correlation between luminosities is 


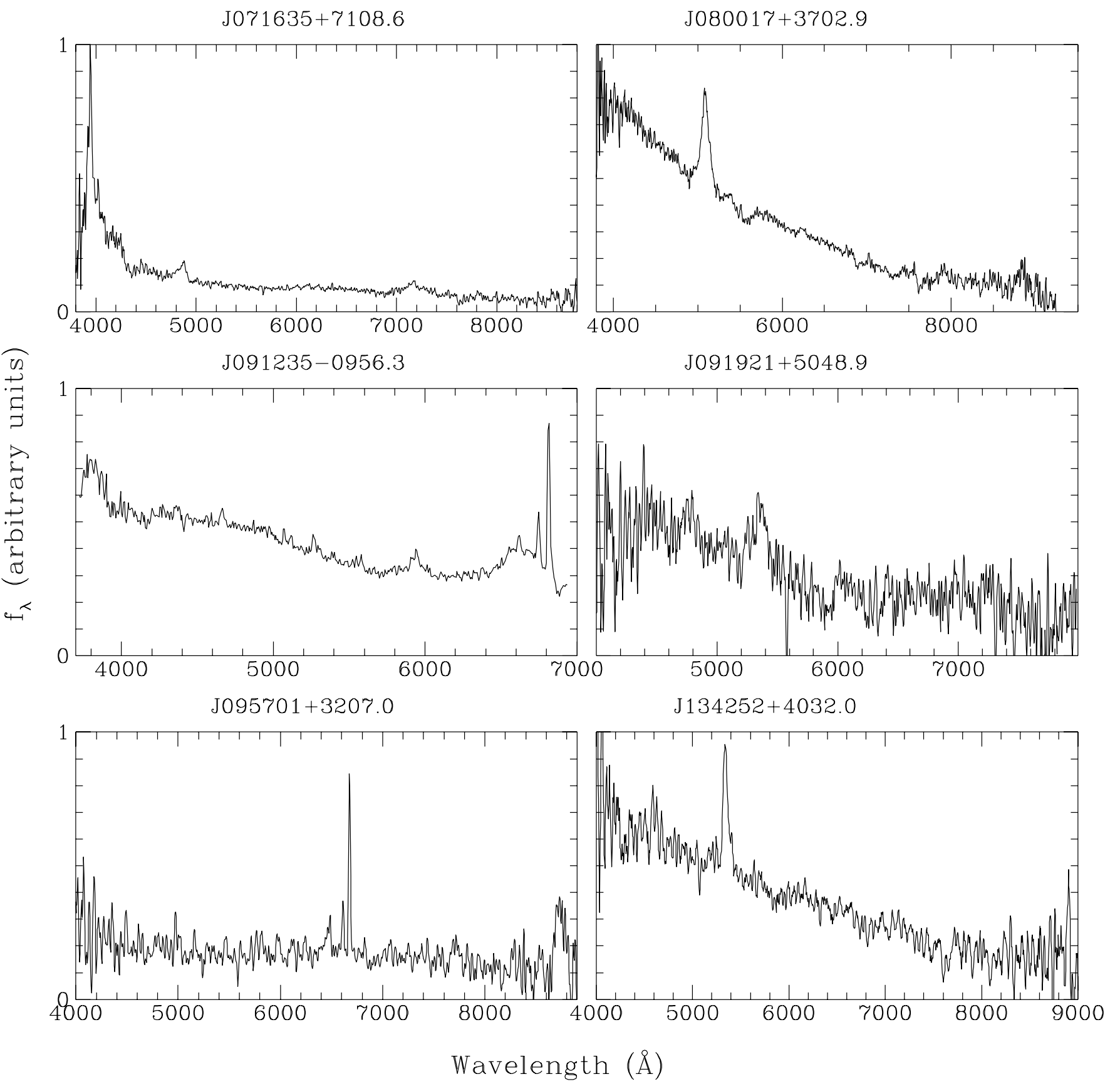

Fig. 2. continued

often observed in flux-limited surveys, due to the artificial correlation between luminosities and redshift. Since we do not see any evidence of a similar correlation between the radio and X-ray fluxes, it is possible that what we observe in Fig. 5 is simply the result of a selection effect. In order to exclude the effect of redshift in the analysis of the correlation bewteen the luminosities, we have used the partial correlation analysis described in Kendall \& Stuart (1979). According to this method, we have computed the correlation coefficient $\left(r_{\mathrm{xr} . \mathrm{z}}\right)$ between $L_{\mathrm{X}}$ and $P_{1.4}$ excluding the dependence to the redshift in the following way:

$r_{\mathrm{xr} . \mathrm{z}}=\frac{r_{\mathrm{xr}}-r_{\mathrm{xz}} r_{\mathrm{rz}}}{\sqrt{1-r_{\mathrm{xz}}^{2}} \sqrt{1-r_{\mathrm{rz}}^{2}}}$

where $r_{\mathrm{xr}}, r_{\mathrm{xz}}$ and $r_{\mathrm{rz}}$ are the Spearman Rank-Order correlation coefficients between $L_{\mathrm{X}} / P_{1.4}, L_{\mathrm{X}} / z$ and $P_{1.4} / z$ respectively. The analysis gives $r_{\mathrm{xr} . \mathrm{z}}=0.09$ corresponding to a probability for the "null hypothesis" (the two luminosities are unrelated) of $\sim 25 \%$. Thus, once excluded the dependence to the redshift, our data do not show any strong 


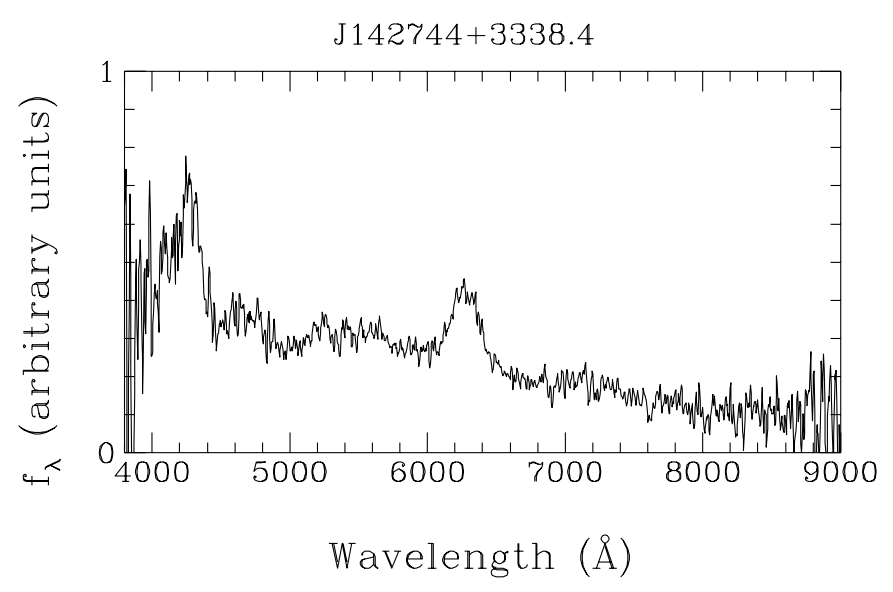

Fig. 2. continued

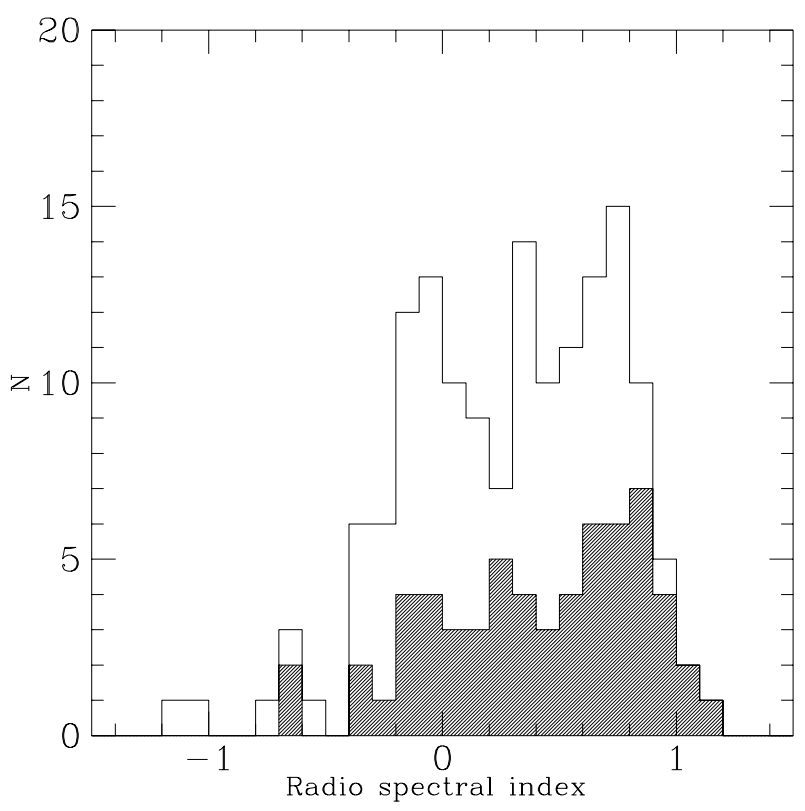

Fig. 3. Distribution of the radio spectral index between $1.4 \mathrm{GHz}$ and $5 \mathrm{GHz}\left(f \propto \nu^{-\alpha}\right)$ for the AGN identified so far in the REX sample and for which a radio flux density at $5 \mathrm{GHz}$ was available. The shaded histogram represents the sources with a flux density at $1.4 \mathrm{GHz}$ larger than $200 \mathrm{mJy}$ and falling in the area of sky covered by the GB6 catalog

evidence of a significant correlation between the two luminosities.

The distribution of X-ray luminosities is similar to that found for the RL AGNs in the EMSS (Della Ceca et al. 1994) and it is peaked at $L_{\mathrm{X}}=510^{45} \mathrm{erg} \mathrm{s}^{-1}$.

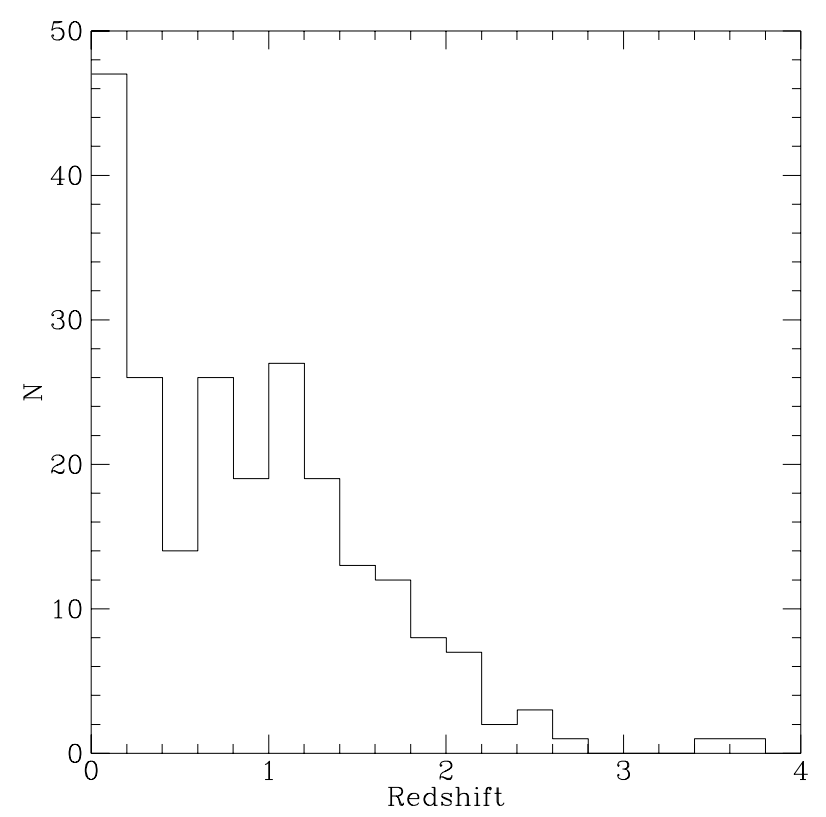

Fig. 4. Redshift distribution of the 226 AGNs found in the REX survey so far

In Fig. 6 we report the Radio-optical $\left(\alpha_{\mathrm{RO}}\right)$ versus the X-ray-optical $\left(\alpha_{\mathrm{OX}}\right)$ spectral indices of the 151 AGNs presented in Fig. 5. These indices are defined in the usual way (e.g. Stocke et al. 1991):

$\alpha_{\mathrm{RO}}=\log \left(S_{5 \mathrm{GHz}} / S_{2500 \mathrm{~A}}\right) / 5.38$

$\alpha_{\mathrm{OX}}=-\log \left(S_{2 \mathrm{keV}} / S_{2500 \mathrm{~A}}\right) / 2.605$

where $S_{5 \mathrm{GHz}}, S_{2500 \AA}$ and $S_{2} \mathrm{keV}$ are the K-corrected monochromatic flux densities at $5 \mathrm{GHz}, 2500 \AA$ and $2 \mathrm{keV}$ respectively. The optical flux densities have been derived from the APM (Automatic Plate Measuring) O (blue) magnitude assuming $\alpha_{\mathrm{O}}=1$, while the monochromatic fluxes at $2 \mathrm{keV}$ have been computed from the X-ray fluxes assuming $\alpha_{\mathrm{X}}=1$. In the radio band we have used the flux density at $5 \mathrm{GHz}$ from GB6 or PMN. About $88 \%$ of the sources have an $\alpha_{\mathrm{RO}} \geq 0.35$, which is the typical limit used to define an object as "radio-loud" (RL, e.g. Della Ceca et al. 1994).

\section{Conclusion}

We have reported the spectra and the main properties of 90 EL objects, 80 of which are newly discovered. Seventyone objects belong to a well defined sample of RadioEmitting X-ray sources, the REX catalog (Caccianiga et al. 1999). The majority of the objects presented here show broad $\left(F W H M>1000 \mathrm{~km} \mathrm{~s}^{-1}\right)$ emission lines in the optical spectrum while only $9 \%$ are type 2 sources, i.e. Narrow Emission line objects. Except for three sources, all the objects presented in this paper are AGNs (QSOs, Seyfert galaxies, emission line radiogalaxies). The sample 
Table 1. Observing setup

\begin{tabular}{|c|c|c|c|}
\hline Telescope/Instrument & Grism name $(\mathrm{g} / \mathrm{mm})$ & $\begin{array}{l}\text { Dispersion } \\
\AA / \text { pixel }\end{array}$ & Observing Period \\
\hline $\mathrm{UH} 88^{\prime \prime}+$ WFGS & blue (400) & 4.2 & 1996 Jan. $14-15$ \\
\hline UH $88^{\prime \prime}+$ WFGS & green $(420)$ & 3.7 & 1996 Aug. 7-11 \\
\hline UNAM $2.1 \mathrm{~m}+\mathrm{BC}$ & $(300)$ & 3.9 & 1996 Dec. 6-10 \\
\hline ESO $2.2 \mathrm{~m}+\mathrm{EFOSC} 2$ & G1 (100) & 13.2 & 1996 Dec. $11-12$ \\
\hline ESO $3.6 \mathrm{~m}+$ EFOSC1 & b300 (300), r300 (300) & $6.3,7.5$ & 1996 Dec. $9-10$ \\
\hline UH $88^{\prime \prime}+$ WFGS & blue (400) & 4.2 & 1997 Mar. 3-5 \\
\hline $\mathrm{UH} 88^{\prime \prime}+$ WFGS & blue (400) & 4.2 & 1998 Feb. 26 - Mar. 1 \\
\hline $\mathrm{UH} 88^{\prime \prime}+$ WFGS & blue (400) & 4.2 & 1998 Oct. $15-18$ \\
\hline
\end{tabular}

Table 2. Journal of observations carried out at the UH $88^{\prime \prime}$ (continued on the next page)

\begin{tabular}{|c|c|c|c|c|c|c|}
\hline Name & NVSS Position (J2000) & $F_{\mathrm{X}}^{a}$ & $S_{1.4}^{b}$ & Set-up ${ }^{c}$ & Date & $\begin{array}{r}\text { Exposure } \\
\text { Time }^{d}\end{array}$ \\
\hline 1REXJ000513-2614.6 & $00 \quad 0513.71-261437.2$ & 1.36 & 37.4 & $420 ; 2.3^{\prime \prime}$ & $08 / 96$ & 1200 \\
\hline 1REXJ001028+2047.8 & $001028.80+204749.4$ & 2.86 & 158.9 & $420 ; 2.3^{\prime \prime}$ & $08 / 96$ & 1200 \\
\hline 1REXJ002031-1510.8 & $002031.06-151049.1$ & 1.93 & 16.6 & $420 ; 2.3^{\prime \prime}$ & 08/96 & 900 \\
\hline 1REXJ002841+0533.0 & $002841.91+053304.0$ & 1.56 & 7.1 & $420 ; 2.3^{\prime \prime}$ & $08 / 96$ & 1200 \\
\hline 1REXJ004052-2902.2 & $00 \quad 4052.42-2902 \quad 15.1$ & 3.95 & 49.5 & $400 ; 1.5^{\prime \prime}$ & $07 / 96$ & 600 \\
\hline 1REXJ004413+0051.6 & $004413.82+005140.7$ & 0.71 & 40.5 & $420 ; 2.3^{\prime \prime}$ & $08 / 96$ & 1800 \\
\hline 1REXJ005924+2703.5 & $005924.40+270332.9$ & 1.21 & 113.4 & $400 ; 1.5^{\prime \prime}$ & $10 / 98$ & 420 \\
\hline 1REXJ011035-1648.5 & $011035.13-164831.3$ & 9.36 & 59.5 & $420 ; 2.3^{\prime \prime}$ & $08 / 96$ & 2100 \\
\hline 1REXJ012210+0931.7 & $0122 \quad 10.66+093144.9$ & 1.74 & 8.9 & $420 ; 2.3^{\prime \prime}$ & $08 / 96$ & 900 \\
\hline 1REXJ012526+0856.5 & $012526.80+085631.1$ & 1.58 & 9.9 & $420 ; 2.3^{\prime \prime}$ & $08 / 96$ & 1800 \\
\hline J013707-2444.7 & $013707.66-244447.8$ & $* 0.53$ & 21.2 & $420 ; 2.3^{\prime \prime}$ & $08 / 96$ & 1800 \\
\hline 1REXJ014318+0228.3 & $0143 \quad 18.58+02 \quad 2820.7$ & 0.45 & 15.8 & $420 ; 2.3^{\prime \prime}$ & $08 / 96$ & 1800 \\
\hline 1REXJ015232-1412.6 & $015232.06-141238.2$ & 2.88 & 745.2 & $420 ; 2.3^{\prime \prime}$ & $08 / 96$ & 900 \\
\hline 1REXJ020857-1003.2 & $020857.04-100316.8$ & 0.42 & 27.0 & $400 ; 1.5^{\prime \prime}$ & $01 / 96$ & 1800 \\
\hline 1REXJ023556+1615.3 & $023556.83+161523.9$ & 5.04 & 129.9 & $400 ; 1.5^{\prime \prime}$ & $10 / 98$ & 900 \\
\hline 1REXJ024613+1056.9 & $024613.81+105656.7$ & 20.10 & 19.9 & $420 ; 2.3^{\prime \prime}$ & 08/96 & 900 \\
\hline J025057-1226.2 & $025057.33-122615.8$ & $*_{2} .30$ & 14.6 & $400 ; 1.5^{\prime \prime}$ & $01 / 96$ & 900 \\
\hline J025929+1925.7 & $025929.65+192544.9$ & $* 2.34$ & 169.9 & $400 ; 1.5^{\prime \prime}$ & $01 / 96$ & 1200 \\
\hline 1REXJ030459+0002.5 & $030459.24+000233.6$ & 3.13 & 124.9 & $400 ; 1.5^{\prime \prime}$ & $01 / 96$ & 960 \\
\hline 1REXJ031958+0355.9 & $031958.82+035556.4$ & 7.20 & 55.7 & $400 ; 1.5^{\prime \prime}$ & $10 / 98$ & 900 \\
\hline J033437-2559.5 & $033437.63-255934.5$ & $* 0.76$ & 28.9 & $420 ; 2.3^{\prime \prime}$ & 08/96 & 1800 \\
\hline 1REXJ034026-2234.9 & $034026.29-223454.2$ & 1.14 & 96.0 & $420 ; 2.3^{\prime \prime}$ & $08 / 96$ & 1200 \\
\hline 1REXJ041322+2343.5 & $041322.50+234335.3$ & 5.46 & 62.3 & $400 ; 1.5^{\prime \prime}$ & $10 / 98$ & 900 \\
\hline 1REXJ041734-1154.5 & $041734.91-115434.2$ & 26.70 & 30.8 & $400 ; 1.5^{\prime \prime}$ & $10 / 98$ & 1590 \\
\hline J053611+6027.3 & $053611.23+602723.5$ & $* 9.80$ & 13.7 & $400 ; 1.5^{\prime \prime}$ & $01 / 96$ & 900 \\
\hline 1REXJ061757+7816.1 & $06 \quad 1757.03+78 \quad 16 \quad 09.0$ & 1.02 & 156.9 & $400 ; 1.5^{\prime \prime}$ & $02 / 98$ & 1800 \\
\hline 1REXJ065154+6955.4 & $065154.56+695526.4$ & 0.97 & 276.3 & $400 ; 1.5^{\prime \prime}$ & $02 / 98$ & 1800 \\
\hline J071635+7108.6 & $071635.45+710838.7$ & $* 0.69$ & 14.6 & $400 ; 1.5^{\prime \prime}$ & $01 / 96$ & 2400 \\
\hline 1REXJ071859+7124.3 & $071859.61+712418.0$ & 0.75 & 182.7 & $400 ; 1.5^{\prime \prime}$ & $01 / 96$ & 1200 \\
\hline 1REXJ073125+6718.7 & $073125.55+671847.4$ & 3.67 & 56.7 & $400 ; 1.5^{\prime \prime}$ & $02 / 98$ & 1200 \\
\hline J080017+3702.9 & $080017.49+370259.8$ & 1.81 & 30.9 & $400 ; 1.5^{\prime \prime}$ & $03 / 97$ & 900 \\
\hline 1REXJ081108+4533.8 & $081108.81+453349.4$ & 1.41 & 81.0 & $400 ; 1.5^{\prime \prime}$ & $02 / 98$ & 1200 \\
\hline 1REXJ082656+6542.5 & $082656.86+654231.9$ & 0.72 & 34.4 & $400 ; 1.5^{\prime \prime}$ & $02 / 98$ & 1800 \\
\hline 1REXJ082733+2637.2 & $082733.76+263716.5$ & 0.56 & 102.4 & $400 ; 1.5^{\prime \prime}$ & $02 / 98$ & 1800 \\
\hline 1REXJ085211+7627.3 & $\begin{array}{lllll}08 & 52 & 11.88+76 & 27 & 18.2\end{array}$ & 1.62 & 191.9 & $400 ; 1.5^{\prime \prime}$ & $01 / 96$ & 900 \\
\hline
\end{tabular}


Table 2. continued

\begin{tabular}{|c|c|c|c|c|c|c|}
\hline Name & NVSS Position (J2000) & $F_{\mathrm{X}}^{a}$ & $S_{1.4}^{b}$ & Set-up ${ }^{c}$ & Date & $\begin{array}{r}\text { Exposure } \\
\text { Time }^{d} \\
\end{array}$ \\
\hline 1REXJ092655-2345.4 & $092655.95-234524.0$ & 2.39 & 83.6 & $400 ; 1.5^{\prime \prime}$ & $02 / 98$ & 1800 \\
\hline J095701+3207.0 & $095701.54+320704.7$ & $* 0.85$ & 69.5 & $400 ; 1.5^{\prime \prime}$ & $01 / 96$ & 1200 \\
\hline 1REXJ102106+4523.4 & $102106.01+452328.5$ & 5.27 & 131.2 & $400 ; 1.5^{\prime \prime}$ & $03 / 97$ & 900 \\
\hline 1REXJ102556+1253.8 & $102556.33+125349.0$ & 2.67 & 539.4 & $400 ; 1.5^{\prime \prime}$ & $02 / 98$ & 1500 \\
\hline 1REXJ103035+5132.5 & $103035.18+513232.9$ & 7.02 & 185.4 & $400 ; 1.5^{\prime \prime}$ & $02 / 98$ & 1800 \\
\hline 1REXJ103206-1400.3 & 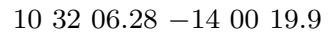 & 1.32 & 196.1 & $400 ; 1.5^{\prime \prime}$ & $03 / 97$ & 1500 \\
\hline 1REXJ121303+3247.6 & $121303.81+324737.0$ & 1.47 & 140.1 & $400 ; 1.5^{\prime \prime}$ & $01 / 96$ & 1200 \\
\hline 1REXJ121815+0744.4 & $1218 \quad 15.55+074428.2$ & 1.03 & 5.1 & $400 ; 1.5^{\prime \prime}$ & $02 / 98$ & 900 \\
\hline 1REXJ123519+6853.6 & $123519.22+685336.8$ & 1.65 & 126.5 & $400 ; 1.5^{\prime \prime}$ & $02 / 98$ & 1200 \\
\hline 1REXJ133714-1319.2 & $13 \quad 37 \quad 14.85-131916.8$ & 2.80 & 114.5 & $400 ; 1.5^{\prime \prime}$ & $02 / 98$ & 1500 \\
\hline 1REXJ134133+3532.8 & $134133.14+353253.7$ & 2.18 & 77.7 & $400 ; 1.5^{\prime \prime}$ & $03 / 97$ & 1800 \\
\hline $\mathrm{J} 134252+4032.0$ & $134252.97+403201.5$ & 4.05 & 152.2 & $400 ; 1.5^{\prime \prime}$ & $01 / 96$ & 600 \\
\hline 1REXJ134606+4859.6 & $134606.12+485936.6$ & 2.18 & 5.2 & $400 ; 1.5^{\prime \prime}$ & $02 / 98$ & 1500 \\
\hline 1REXJ135409-0141.8 & $135409.97-014150.4$ & 1.76 & 39.1 & $400 ; 1.5^{\prime \prime}$ & $02 / 98$ & 1500 \\
\hline 1REXJ140653+3433.6 & $140653.86+343337.2$ & 1.77 & 169.8 & $400 ; 1.5^{\prime \prime}$ & $03 / 97$ & 900 \\
\hline 1REXJ141628+1242.2 & $141628.64+124213.5$ & 7.36 & 110.6 & $400 ; 1.5^{\prime \prime}$ & & 2400 \\
\hline $\mathrm{J} 142744+3338.4$ & $142744.44+333828.6$ & $*^{*} 0.83$ & 16.3 & $400 ; 1.5^{\prime \prime}$ & $01 / 96$ & 1500 \\
\hline 1REXJ144544-2445.7 & $144544.21-244542.1$ & 4.57 & 210.5 & $400 ; 1.5^{\prime \prime}$ & $02 / 98$ & 1800 \\
\hline 1REXJ152548+5828.8 & $152548.20+582851.4$ & 0.69 & 109.3 & $400 ; 1.5^{\prime \prime}$ & $02 / 98$ & 1200 \\
\hline 1REXJ213248-0219.8 & $213248.21-021950.5$ & 7.80 & 29.1 & $400 ; 1.5^{\prime \prime}$ & $10 / 98$ & 900 \\
\hline 1REXJ220451-1815.5 & $\begin{array}{llllll}22 & 04 & 51.82 & -18 & 15 & 35.4\end{array}$ & 10.40 & 38.5 & $400 ; 1.5^{\prime \prime}$ & $10 / 98$ & 1800 \\
\hline 1REXJ223313+3405.0 & $223313.05+340501.0$ & 3.42 & 35.3 & $400 ; 1.5^{\prime \prime}$ & $10 / 98$ & 900 \\
\hline 1REXJ230311-0859.3 & $\begin{array}{llllll}23 & 03 & 11.03 & -08 & 59 & 19.7\end{array}$ & 0.54 & 33.6 & $400 ; 1.5^{\prime \prime}$ & $10 / 98$ & 480 \\
\hline 1REXJ235029-2620.7 & $235029.61-262046.1$ & 8.86 & 11.1 & $400 ; 1.5^{\prime \prime}$ & $10 / 98$ & 600 \\
\hline 1REXJ235139-2605.0 & $\begin{array}{llllll}23 & 51 & 39.37 & -26 & 05 & 02.7\end{array}$ & 40.20 & 20.1 & $400 ; 1.5^{\prime \prime}$ & $10 / 98$ & 900 \\
\hline
\end{tabular}

${ }^{a}$ X-ray fluxes, corrected for Galactic absorption, in the $0.5-2.0 \mathrm{keV}$ band in units of $10^{-13} \mathrm{erg} \mathrm{s}^{-1} \mathrm{~cm}^{-2}$; fluxes with an asterisk come from the 1RXP catalog (see text for details).

${ }^{b}$ Radio flux densities at $1.4 \mathrm{GHz}$ in $\mathrm{mJy}$.

${ }^{c}$ First column $=$ Grism $(400=$ Grism $400 \mathrm{l} / \mathrm{mm}, 420=$ Grism $420 \mathrm{l} / \mathrm{mm}) ;$ second column $=$ slit width.

${ }^{d}$ Exposure time in seconds.

Table 3. Journal of observations carried out at the ESO telescopes

\begin{tabular}{rlrrlr} 
Name & NVSS Position (J2000) & $F_{\mathrm{X}}^{a}$ & $S_{1.4}^{b}$ & Set-up $^{c}$ & $\begin{array}{r}\text { Exposure } \\
\text { Time }^{d}\end{array}$ \\
\hline J001341-3009.4 & $001341.24-300926.6$ & 0.43 & 223.0 & $2.2 \mathrm{~m} ; \mathrm{G} 1$ & 1200 \\
1REXJ005229-3743.8 & $005229.01-374350.1$ & 0.52 & 30.2 & $3.6 \mathrm{~m} ; \mathrm{b} 300$ & 600 \\
J014716-0008.2 & $014716.08-000817.7$ & 1.14 & 5.0 & $3.6 \mathrm{~m} ; \mathrm{b} 300$ & 2400 \\
1REXJ015429-1346.6 & $015429.41-134636.4$ & 0.46 & 74.3 & $3.6 \mathrm{~m} ; \mathrm{b} 300$ & 1200 \\
J033953-2321.6 & $033953.54-232136.7$ & 0.33 & 36.6 & $3.6 \mathrm{~m} ; \mathrm{b} 300, \mathrm{r} 300$ & 840,300 \\
1REXJ035348-1020.2 & $035348.77-102014.3$ & 0.70 & 19.1 & $2.2 \mathrm{~m} ; \mathrm{G} 1$ & 1200 \\
J040741-3102.7 & $040741.85-310246.5$ & 0.51 & 49.3 & $2.2 \mathrm{~m} ; \mathrm{G} 1$ & 720 \\
1REXJ041405-1224.2 & $041405.96-122417.0$ & 3.06 & 93.8 & $2.2 \mathrm{~m} ; \mathrm{G} 1$ & 1200 \\
1REXJ043851-2241.7 & $043851.91-224144.3$ & 0.83 & 217.0 & $2.2 \mathrm{~m} ; \mathrm{G} 1$ & 720 \\
1REXJ044105-1616.1 & $044105.05-161607.1$ & 1.26 & 45.1 & $3.6 \mathrm{~m} ; \mathrm{b} 300, \mathrm{r} 300$ & 1200,600 \\
1REXJ044910-2015.4 & $044910.33-201524.7$ & 1.30 & 10.7 & $3.6 \mathrm{~m} ; \mathrm{b} 300$ & 1500 \\
1REXJ045756-2237.7 & $045756.98-223745.8$ & 2.70 & 11.5 & $2.2 \mathrm{~m} ; \mathrm{G} 1$ & 1200 \\
1REXJ053628-3401.1 & $053628.46-340111.0$ & 7.33 & 652.6 & $2.2 \mathrm{~m} ; \mathrm{G} 1$ & 900 \\
1REXJ054129-3427.7 & $054129.75-342743.1$ & 0.73 & 33.1 & $2.2 \mathrm{~m} ; \mathrm{G} 1$ & 1080 \\
1REXJ055722-1414.7 & $055722.80-141442.4$ & 1.74 & 5.5 & $3.6 \mathrm{~m} ; \mathrm{b} 300$ & 1200 \\
1REXJ062437-1824.1 & $062437.43-182408.4$ & 6.02 & 6.5 & $3.6 \mathrm{~m} ; \mathrm{b} 300$ & 1260 \\
J063114-2257.3 & $063114.18-225719.8$ & $* 1.64$ & 19.8 & $3.6 \mathrm{~m} ; \mathrm{b} 300$ & 300 \\
J063155-2250.4 & $063155.08-225024.2$ & $* 1.95$ & 77.1 & $3.6 \mathrm{~m} ; \mathrm{b} 300$ & 1500 \\
1REXJ085119+1358.4 & $085119.81+135825.7$ & 2.82 & 22.4 & $2.2 \mathrm{~m} ; \mathrm{G} 1$ & 720 \\
1REXJ085312+1358.8 & $085312.21+135853.3$ & 0.46 & 17.4 & $2.2 \mathrm{~m} ; \mathrm{G} 1$ & 1500 \\
1REXJ090015-2817.9 & $090015.43-281758.6$ & 2.61 & 512.1 & $2.2 \mathrm{~m} ; \mathrm{G} 1$ & 300 \\
J091235-0956.3 & $091235.74-095621.8$ & 5.35 & 57.1 & $3.6 \mathrm{~m} ; \mathrm{b} 300$ & 1260 \\
1REXJ105027-2816.0 & $105027.31-281604.4$ & 0.94 & 19.1 & $3.6 \mathrm{~m} ; \mathrm{b} 300$ & 600 \\
1REXJ10744-3043.5 & $110744.07-304335.6$ & 1.44 & 351.5 & $3.6 \mathrm{~m} ; \mathrm{b} 300$ & \\
\hline
\end{tabular}

a See Table 2.

${ }^{b}$ See Table 2 .

${ }^{c}$ First column $=$ telescope; second column $=$ Grism. The slit width is always $1.5^{\prime \prime}$.

$d$ See Table 2 . 
Table 4. Journal of observations carried out at the UNAM $2.1 \mathrm{~m}$

\begin{tabular}{rcrrrr} 
Name & NVSS Position (J2000) & $F_{\mathrm{X}}^{a}$ & $S_{1.4}^{b}$ & Slit width $^{c}$ & $\begin{array}{r}\text { Exposure } \\
\text { Time }^{d}\end{array}$ \\
\hline J000640+2042.7 & $000640.05+204245.0$ & 0.50 & 80.5 & 1.6 & 4800 \\
1REXJ022840-0935.2 & $022840.81-093514.6$ & 8.89 & 8.5 & 1.6 & 1800 \\
1REXJ044754-0322.7 & $044754.76-032243.2$ & 10.80 & 87.3 & 1.6 & 900 \\
1REXJ081041+0810.0 & $081041.43+081000.5$ & 3.33 & 198.7 & 1.6 & 3900 \\
J091921+5048.9 & $091921.77+504855.4$ & $* 4.77$ & 46.5 & 1.6 & 2220 \\
1REXJ101238+5242.4 & $101238.56+524225.2$ & 3.74 & 8.1 & 2.4 & 3000 \\
\hline
\end{tabular}

${ }^{a}$ see Table 2.

${ }^{b}$ see Table 2 .

${ }^{c}$ Slit width in arcseconds.

${ }^{d}$ see Table 2 .

Table 5. Emission Line AGNs identified in the REX survey (continued on the next page)

\begin{tabular}{|c|c|c|c|c|c|c|c|}
\hline Name & Class & $z$ & $\mathrm{C}^{a}$ & $\log L_{\mathrm{X}}^{b}$ & $\log P_{1.4}^{c}$ & Emission Lines & Absorption Lines \\
\hline 1REXJ000513-2614.6 & $\mathrm{B}$ & 0.32 & $\mathrm{~F}$ & 43.92 & $32.21^{*}$ & $\mathrm{H} \delta, \mathrm{H} \gamma, \mathrm{H} \beta,[\mathrm{OIII}], \mathrm{H} \alpha$ & \\
\hline 1REXJ001028+2047.8 & B & 0.60 & $\mathrm{~F}$ & 44.88 & 33.46 & $\mathrm{MgII},[\mathrm{OII}], \mathrm{H} \delta, \mathrm{H} \gamma, \mathrm{H} \beta,[\mathrm{OIII}]$ & \\
\hline 1REXJ002031-1510.8 & B & 0.59 & $\mathrm{~F}$ & 44.69 & $32.26^{*}$ & $\mathrm{MgII},[\mathrm{OII}], \mathrm{H} \gamma, \mathrm{H} \beta,[\mathrm{OIII}]$ & \\
\hline 1REXJ002841+0533.0 & B & 1.43 & $\mathrm{~T}$ & 45.58 & $32.33^{*}$ & MgII? & \\
\hline 1REXJ004052-2902.2 & B & 0.265 & $\mathrm{~F}$ & 44.19 & $32.15^{*}$ & $\mathrm{H} \delta, \mathrm{H} \gamma, \mathrm{H} \beta,[\mathrm{OIII}], \mathrm{H} \alpha$ & \\
\hline 1REXJ004413+0051.6 & B & 0.93 & $\mathrm{~F}$ & 44.75 & $33.22^{*}$ & $\mathrm{MgII},[\mathrm{NeV}]$ & \\
\hline 1REXJ005229-3743.8 $8^{(1)}$ & B & 1.60 & $\mathrm{~F}$ & 45.23 & $33.45^{*}$ & $\mathrm{CIV}, \mathrm{CIII}]$ & \\
\hline 1REXJ005924+2703.5 & $\mathrm{N}$ & 0.045 & $\mathrm{~F}$ & 42.05 & 31.00 & $\mathrm{H} \beta,[\mathrm{OIII}], \mathrm{H} \alpha /[\mathrm{NII}],[\mathrm{SII}]$ & \\
\hline 1REXJ011035-1648.5 (2) & B & 0.783 & $\mathrm{~F}$ & 45.68 & 33.56 & $\mathrm{MgII}, \mathrm{H} \delta, \mathrm{H} \gamma, \mathrm{H} \beta$ & \\
\hline 1REXJ012210+0931.7 & B & 0.33 & $\mathrm{~F}$ & 44.05 & $31.47^{*}$ & {$[\mathrm{NeV}],[\mathrm{OII}], \mathrm{H} \gamma, \mathrm{H} \beta,[\mathrm{OIII}], \mathrm{H} \alpha$} & \\
\hline 1REXJ012526+0856.5 & B & 0.895 & $\mathrm{~T}$ & 45.06 & $32.25^{*}$ & MgII? & \\
\hline 1REXJ014318+0228.3 & B & 0.83 & $\mathrm{~T}$ & 44.43 & $32.52^{*}$ & MgII? & \\
\hline 1REXJ015232-1412.6 & B & 1.35 & $\mathrm{~F}$ & 45.78 & 35.00 & CIII],MgII, [OII] & \\
\hline 1REXJ015429-1346.6 & B & 1.01 & $\mathrm{~F}$ & 44.65 & 33.54 & CIII],MgII & \\
\hline 1REXJ020857-1003.2 & B & 1.74 & $\mathrm{~F}$ & 45.22 & $33.49^{*}$ & CIV, $\mathrm{CIII}]$ & \\
\hline 1REXJ022840-0935.2 & $\mathrm{N}$ & 0.068 & $\mathrm{~F}$ & 43.28 & $30.19^{*}$ & $\mathrm{H} \gamma, \mathrm{H} \beta,[\mathrm{OIII}],[\mathrm{OI}], \mathrm{H} \alpha,[\mathrm{SII}]$ & \\
\hline 1REXJ023556+1615.3 & B & 0.256 & $\mathrm{~F}$ & 44.26 & 32.57 & $\mathrm{H} \delta, \mathrm{H} \gamma, \mathrm{H} \beta,[\mathrm{OIII}], \mathrm{H} \alpha$ & \\
\hline 1REXJ024613+1056.9 & B & 0.357 & $\mathrm{~F}$ & 45.19 & 31.84 & $\mathrm{H} \delta, \mathrm{H} \gamma, \mathrm{FeII}, \mathrm{H} \beta,[\mathrm{OIII}], \mathrm{H} \alpha$ & \\
\hline 1REXJ030459+0002.5 $5^{(3)}$ & B & 0.564 & $\mathrm{~F}$ & 44.86 & 33.33 & $\mathrm{MgII},[\mathrm{NeV}],[\mathrm{OII}], \mathrm{H} \delta, \mathrm{H} \beta,[\mathrm{OIII}]$ & \\
\hline 1REXJ031958+0355.9 & B & 0.813 & $\mathrm{~F}$ & 45.61 & 33.23 & $\mathrm{MgII}, \mathrm{H} \gamma$ & \\
\hline 1REXJ034026-2234.9 & B & 1.68 & $\mathrm{~F}$ & 45.67 & 34.41 & CIII],FeII,MgII & \\
\hline 1REXJ035348-1020.2 & B & 0.87 & $\mathrm{~F}$ & 44.67 & $32.67^{*}$ & $\mathrm{CIII}], \mathrm{FeII}, \mathrm{MgII}, \mathrm{H} \beta$ & \\
\hline 1REXJ041322+2343.5 & B & 0.309 & $\mathrm{~F}$ & 44.49 & 32.41 & FeII,H $\gamma, \mathrm{H} \beta,[\mathrm{OIII}], \mathrm{H} \alpha$ & \\
\hline 1REXJ041405-1224.2 $2^{(4)}$ & $\mathrm{B}$ & 0.570 & $\mathrm{~F}$ & 44.87 & 33.27 & $\mathrm{MgII},[\mathrm{NeV}], \mathrm{H} \delta, \mathrm{H} \gamma, \mathrm{H} \beta,[\mathrm{OIII}]$ & \\
\hline 1REXJ041734-1154.5 & $\mathrm{N}$ & 0.44 & $\mathrm{~F}$ & 45.53 & $32.38^{*}$ & [OII] & CaII H\&K \\
\hline 1REXJ043851-2241.7 & $\mathrm{B}$ & 1.66 & $\mathrm{~F}$ & 45.52 & 34.83 & CIV,CIII],MgII & \\
\hline 1REXJ044105-1616.1 & $\mathrm{B}$ & 0.408 & $\mathrm{~F}$ & 44.13 & $32.53^{*}$ & $\mathrm{MgII},[\mathrm{OII}],[\mathrm{OIII}], \mathrm{H} \alpha$ & CaII H\&K \\
\hline 1REXJ044754-0322.7 $7^{(5)}$ & $\mathrm{B}$ & 0.773 & $\mathrm{~F}$ & 45.73 & 33.48 & $\mathrm{FeII}, \mathrm{MgII}, \mathrm{FeII},[\mathrm{OII}], \mathrm{H} \delta, \mathrm{H} \gamma$ & \\
\hline 1REXJ044910-2015.4 & $\mathrm{B}$ & 1.04 & $\mathrm{~F}$ & 45.14 & $32.40^{*}$ & CIII],MgII & \\
\hline 1REXJ045756-2237.7 & $\mathrm{B}$ & 0.12 & $\mathrm{~F}$ & 43.28 & $30.80^{*}$ & $\mathrm{H} \alpha$ & CaII $\mathrm{H} \& \mathrm{~K}, \mathrm{G}, \mathrm{MgI}$ \\
\hline 1REXJ053628-3401.1 & B & 0.684 & $\mathrm{~F}$ & 45.43 & 34.15 & $\mathrm{MgII},[\mathrm{NeV}], \mathrm{H} \delta, \mathrm{H} \gamma, \mathrm{H} \beta,[\mathrm{OIII}]$ & \\
\hline 1REXJ054129-3427.7 & B & 1.60 & $\mathrm{~F}$ & 45.38 & $33.40^{*}$ & SiIV/OIV],CIV,CIII],MgI & \\
\hline 1REXJ055722-1414.7 & B & 0.358 & $\mathrm{~F}$ & 44.13 & $31.26^{*}$ & $\mathrm{MgII},[\mathrm{OII}],[\mathrm{NeIII}], \mathrm{H} \delta, \mathrm{H} \gamma, \mathrm{H} \beta,[\mathrm{OIII}]$ & \\
\hline
\end{tabular}

contains two high redshift $(z=3.47$ and 3.49$)$ radio-loud AGNs.

We have then studied the general properties of the 226 AGNs discovered so far in the REX sample, including sources identified from literature. We have analyzed their radio properties (compactness and slope) as deduced from the NVSS data. On average, we select a similar number of SS and FS radio sources despite the fact that the selection has been done by using a relatively low frequency $(1.4 \mathrm{GHz})$ radio catalog. At this frequency, we would have expected a dominance of SS AGNs. The large number of
FS AGNs found in this sample is probably due to the presence of the X-ray constraint that favors the selection of compact FS sources. On the other side, the presence of a radio limit favors the selection of $\mathrm{RL}$ AGNs. In fact, the majority of these sources $(\sim 88 \%)$ is radio loud while only a small percentage $(\sim 10 \%$, Della Ceca et al. 1994) of such objects is expected in a purely X-ray selected survey.

Acknowledgements. We are grateful to the staff at the UH (Hawaii, U.S.A.), ESO (La Silla, Chile) and UNAM (San Pedro Martir, Mexico) telescopes for their support during the observing runs. This research was partially supported by the Italian 
Table 5. continued

\begin{tabular}{|c|c|c|c|c|c|c|c|}
\hline Name & Class & $z$ & $\mathrm{C}^{a}$ & $\log L_{\mathrm{X}}^{b}$ & $\log P_{1.4}^{c}$ & Emission Lines & Absorption Lines \\
\hline 1REXJ061757+7816.1 & $\mathrm{B}$ & 1.43 & $\mathrm{~F}$ & 45.39 & 34.34 & CIII],MgII & \\
\hline 1REXJ062437-1824.1 & B & 1.89 & $\mathrm{~F}$ & 46.48 & $32.40^{*}$ & CIV,CIII] & \\
\hline 1REXJ065154+6955.4 & B & 1.36 & $\mathrm{~F}$ & 45.35 & 34.64 & CIII],MgII & \\
\hline 1REXJ071859+7124.3 ${ }^{(6)}$ & B & 1.408 & $\mathrm{~F}$ & 45.28 & 34.60 & CIII], MgII & \\
\hline 1REXJ073125+6718.7 & B & 0.17 & $\mathrm{~F}$ & 43.74 & 31.78 & $\mathrm{H} \gamma, \mathrm{H} \beta,[\mathrm{OIII}], \mathrm{H} \alpha$ & CaII K \\
\hline 1REXJ081041+0810.0 & B & 0.391 & $\mathrm{~F}$ & 44.51 & 33.20 & {$[\mathrm{OII}],[\mathrm{NeIII}], \mathrm{H} \gamma, \mathrm{H} \beta,[\mathrm{OIII}]$} & \\
\hline 1REXJ081108+4533.8 & B & 1.02 & $\mathrm{~F}$ & 45.15 & 33.50 & FeII,MgII,[OII],[NeIII],H $\delta, \mathrm{H} \gamma$ & \\
\hline 1REXJ082656+6542.5 & B & 0.956 & $\mathrm{~F}$ & 44.79 & 33.41 & $\mathrm{MgII}, \mathrm{H} \gamma$ & \\
\hline 1REXJ082733+2637.2(7) & B & 0.69 & $\mathrm{~F}$ & 44.34 & 33.52 & $\mathrm{MgII},[\mathrm{OII}], \mathrm{H} \gamma,[\mathrm{OIII}]$ & \\
\hline 1REXJ085120+1358.3 & B & 0.95 & $\mathrm{~F}$ & 45.37 & 33.06 & $\mathrm{CIII}], \mathrm{MgII},[\mathrm{NeV}], \mathrm{H} \delta$ & \\
\hline 1REXJ085211+7627.3 & B & 1.127 & $\mathrm{~F}$ & 45.32 & 34.34 & CIII],MgII & \\
\hline 1REXJ085312+1358.8 & B & 1.16 & $\mathrm{~F}$ & 44.81 & $33.05^{*}$ & $\mathrm{CIII}], \mathrm{MgII}$ & \\
\hline 1REXJ090015-2817.9 & B & 0.89 & $\mathrm{~F}$ & 45.29 & 34.46 & $\mathrm{CIII}], \mathrm{MgII},[\mathrm{NeV}],[\mathrm{OII}],[\mathrm{NeIII}], \mathrm{H} \gamma, \mathrm{H} \beta,[\mathrm{OIII}]$ & \\
\hline 1REXJ092655-2345.4 & B & 1.17 & $\mathrm{~F}$ & 45.54 & 33.72 & $\mathrm{CIII}] ?, \mathrm{MgII},[\mathrm{NeV}] ?$ & \\
\hline 1REXJ101238+5242.4 & B & 0.129 & $\mathrm{~F}$ & 43.48 & $30.73^{*}$ & {$[\mathrm{OII}], \mathrm{H} \delta, \mathrm{H} \gamma, \mathrm{H} \beta,[\mathrm{OIII}],[\mathrm{OI}], \mathrm{H} \alpha,[\mathrm{SII}]$} & MgI,NaID \\
\hline 1REXJ102106+4523.4 ${ }^{(9)}$ & B & 0.364 & $\mathrm{~F}$ & 44.63 & 32.94 & $\mathrm{H} \gamma, \mathrm{H} \beta,[\mathrm{OIII}], \mathrm{H} \alpha$ & \\
\hline 1REXJ102556+1253.8 ${ }^{(10)}$ & B & 0.66 & $\mathrm{~T}$ & 44.95 & 34.00 & $\mathrm{MgII} ?,[\mathrm{OII}] ?, \mathrm{H} \beta$ ? & \\
\hline 1REXJ103035+5132.5 & $\mathrm{B}$ ? & 0.518 & $\mathrm{~F}$ & 45.12 & 33.40 & {$[\mathrm{OII}], \mathrm{H} \gamma, \mathrm{H} \beta$} & CaII K \\
\hline 1REXJ103206-1400.3(11) & B & 1.059 & $\mathrm{~T}$ & 45.17 & 34.00 & MgII?,[OIII]? & \\
\hline 1REXJ105027-2816.0 & $\mathrm{B}$ & 0.41 & $\mathrm{~F}$ & 44.01 & $32.11^{*}$ & $\mathrm{MgII},[\mathrm{NeV}],[\mathrm{OII}], \mathrm{H} \gamma ?, \mathrm{H} \beta$ & CaII H\&K \\
\hline 1REXJ110744-3043.5 & B & 0.74 & $\mathrm{~F}$ & 44.81 & 34.02 & MgII, [NeV],[NeIII] & \\
\hline 1REXJ121303+3247.6 & B & 2.507 & $\mathrm{~F}$ & 46.26 & 35.10 & Ly $\alpha, C I V, C I I I]$ & \\
\hline 1REXJ121815+0744.4 & $\mathrm{N}$ & 0.155 & $\mathrm{~F}$ & 43.10 & $30.62^{*}$ & {$[\mathrm{OIII}], \mathrm{H} \alpha,[\mathrm{SII}]$} & \\
\hline 1REXJ123519+6853.6 & B & 1.14 & $\mathrm{~F}$ & 45.35 & 34.05 & CIII]?,MgII & \\
\hline 1REXJ133714-1319.2 & B & 3.47 & $\mathrm{~F}$ & 46.79 & 35.24 & Ly $\alpha, \mathrm{OI}, \mathrm{SiIV} / \mathrm{OIV}], \mathrm{CIV}, \mathrm{CIII}]$ & \\
\hline 1REXJ134133+3532.8 & B & 0.783 & $\mathrm{~F}$ & 45.05 & 33.36 & $\mathrm{MgII},[\mathrm{OII}], \mathrm{H} \gamma, \mathrm{H} \beta,[\mathrm{OIII}]$ & \\
\hline 1REXJ134606+4859.6 & B & 0.187 & $\mathrm{~F}$ & 43.60 & $30.82^{*}$ & $\mathrm{H} \alpha$ & CaII H\&K \\
\hline 1REXJ135409-0141.8 & B & 0.98 & $\mathrm{~F}$ & 45.20 & $33.25^{*}$ & $\mathrm{MgII},[\mathrm{OII}]$ & \\
\hline 1REXJ140653+3433.6 & B & 2.56 & $\mathrm{~F}$ & 46.28 & 34.76 & Ly $\alpha, \operatorname{SiIV} / \mathrm{OIV}], \mathrm{CIV}, \mathrm{CIII}]$ & \\
\hline 1REXJ141628+1242.2 & B & 0.33 & $\mathrm{~F}$ & 44.68 & 32.74 & $\mathrm{H} \gamma, \mathrm{H} \beta,[\mathrm{OIII}], \mathrm{H} \alpha$ & \\
\hline 1REXJ144544-2445.7 & $\mathrm{B}$ & 0.317 & $\mathrm{~F}$ & 44.43 & 32.95 & $\mathrm{H} \delta, \mathrm{H} \gamma, \mathrm{H} \beta,[\mathrm{OIII}], \mathrm{H} \alpha$ & \\
\hline 1REXJ152548+5828.8 & $\mathrm{B}$ ? & 0.309 & $\mathrm{~F}$ & 43.59 & 32.70 & {$[\mathrm{OIII}], \mathrm{H} \alpha$} & CaII H\&K,G \\
\hline 1REXJ213248-0219.8 & B & 0.103 & $\mathrm{~F}$ & 43.60 & $31.11^{*}$ & {$[\mathrm{NeIII}] ?, \mathrm{H} \delta, \mathrm{H} \gamma, \mathrm{H} \beta,[\mathrm{OIII}], \mathrm{H} \alpha$} & \\
\hline 1REXJ220451-1815.5 & $\mathrm{N}$ & 0.21 & $\mathrm{~T}$ & 44.39 & $31.86^{*}$ & [OII]?,[OIII]? & \\
\hline 1REXJ223313+3405.0 & $\mathrm{B}$ & 0.63 & $\mathrm{~F}$ & 45.01 & 32.76 & $\mathrm{MgII},[\mathrm{NeV}],[\mathrm{OII}],[\mathrm{NeIII}], \mathrm{H} \gamma, \mathrm{H} \beta,[\mathrm{OIII}]$ & \\
\hline 1REXJ230311-0859.3 ${ }^{(12)}$ & $\mathrm{N} ?$ & 0.024 & $\mathrm{~F}$ & 41.14 & $29.92^{*}$ & $\mathrm{H} \alpha$ & MgI,NaID \\
\hline 1REXJ235029-2620.7 & B & 0.217 & $\mathrm{~F}$ & 44.35 & $31.26^{*}$ & {$[\mathrm{OII}],[\mathrm{NeIII}], \mathrm{H} \delta, \mathrm{H} \beta,[\mathrm{OIII}], \mathrm{H} \alpha$} & \\
\hline 1REXJ235139-2605.0 & $\mathrm{N}$ & 0.233 & $\mathrm{~F}$ & 45.08 & $31.62^{*}$ & {$[\mathrm{OII}], \mathrm{H} \beta,[\mathrm{OIII}],[\mathrm{OI}], \mathrm{H} \alpha,[\mathrm{SII}]$} & \\
\hline
\end{tabular}

${ }^{a}$ Redshift confidence: $\mathrm{F}=$ firm, $\mathrm{T}=$ tentative.

${ }^{b}$ De-absorbed (Galactic) X-ray luminosity $\left(0.5-2.0 \mathrm{keV}\right.$ band) in erg s${ }^{-1}$.

${ }^{c}$ Monochromatic radio luminosity at $1.4 \mathrm{GHz}$ in $\mathrm{erg} \mathrm{s}^{-1} \mathrm{~Hz}^{-1}$. An asterisk indicates that the luminosity has been computed assuming a lower limit on the radio spectral index (see text for details);

Notes:

(1) Also in Iovino et al. (1996) $(z=2.25$ based on low dispersion prism observations).

(2) Also in Perlman et al. (1998) $(z=0.78)$.

(3) Also in Perlman et al. (1998) $(z=0.563)$.

(4) Also in Perlman et al. (1998) $(z=0.569)$.

(5) Also in Perlman et al. (1998) $(z=0.774)$.

(6) Also in Puchnarewicz et al. (1997) $(z=1.419)$.

(7) Also in Puchnarewicz et al. (1997) $(z=0.692)$.

(8) Also in Perlman et al. (1998) $(z=0.894)$.

(9) Also in Laurent-Muehleisen et al. (1998) $(z=0.364)$.

(10) Also in Perlman et al. (1998) $(z=0.663)$.

(11) Also in Perlman et al. (1998) $(z=1.039)$.

(12) Also in Da Costa et al. (1998) $(z=0.02413)$. 
Table 6. Emission Line AGNs not included in the final version of the REX catalog

\begin{tabular}{|c|c|c|c|c|c|c|c|}
\hline Name & Class & $z$ & $\mathrm{C}^{a}$ & $\log L_{\mathrm{X}}^{b}$ & $\log P_{1.4}^{c}$ & Emission Lines & Absorption Lines \\
\hline J000640+2042.7 & B & 1.00 & $\mathrm{~T}$ & 44.70 & 33.78 & MgII? & \\
\hline J001341-3009.4 & B & 1.11 & $\mathrm{~F}$ & 44.76 & 34.35 & CIII],MgII & \\
\hline $\mathrm{J} 013707-2444.7^{(1)}$ & B & 1.05 & $\mathrm{~T}$ & 44.76 & $32.89^{*}$ & MgII? & \\
\hline J014716-0008.2 & $\mathrm{N}$ & 0.466 & $\mathrm{~F}$ & 44.22 & $31.41^{*}$ & {$[\mathrm{OII}],[\mathrm{NeIII}], \mathrm{H} \gamma$} & \\
\hline J025057-1226.2 & $\mathrm{B}$ & 1.004 & $\mathrm{~T}$ & 45.35 & $32.60^{*}$ & MgII? & \\
\hline J025929+1925.7 & B & 0.545 & $\mathrm{~F}$ & 44.69 & 33.37 & MgII,FeII,[NeV],H $\beta$ & \\
\hline J033437-2559.5 & B & 1.16 & $\mathrm{~T}$ & 45.03 & $33.19^{*}$ & MgII? & \\
\hline J033953-2321.6 & B & 3.49 & $\mathrm{~F}$ & 46.87 & $34.44^{*}$ & OVI, Ly $\alpha / \mathrm{NV}, \mathrm{OI}, \mathrm{SiIV} / \mathrm{OIV}], \mathrm{CIV}, \mathrm{CIII}]$ & \\
\hline J040741-3102.7 & B & 1.40 & $\mathrm{~F}$ & 45.07 & $33.58^{*}$ & CIV, CIII], MgII & \\
\hline $\mathrm{J} 053611+6027.3$ & $\mathrm{~B}$ & 0.07 & $\mathrm{~F}$ & 43.35 & $30.45^{*}$ & {$[\mathrm{OIII}],[\mathrm{OI}], \mathrm{H} \alpha,[\mathrm{SII}]$} & \\
\hline J063114-2257.3 & $\mathrm{B}$ & 0.86 & $\mathrm{~T}$ & 45.03 & $32.68^{*}$ & MgII? & \\
\hline J063155-2250.4 & $\mathrm{B}$ & 0.589 & $\mathrm{~F}$ & 44.70 & $33.18^{*}$ & $\mathrm{MgII},[\mathrm{OII}]$ & \\
\hline J071635+7108.6 & B & 1.553 & $\mathrm{~F}$ & 45.32 & $33.20^{*}$ & CIV,OIII],CIII],MgII & \\
\hline $\mathrm{J} 080017+3702.9$ & $\mathrm{~B}$ & 0.819 & $\mathrm{~F}$ & 45.02 & 32.88 & $\mathrm{MgII}, \mathrm{FeII},[\mathrm{NeV}], \mathrm{H} \gamma, \mathrm{H} \beta$ & \\
\hline J091235-0956.3 & B & 0.361 & $\mathrm{~F}$ & 44.63 & $32.55^{*}$ & $\mathrm{MgII},[\mathrm{NeV}],[\mathrm{NeIII}], \mathrm{H} \gamma, \mathrm{H} \beta,[\mathrm{OIII}]$ & \\
\hline J091921+5048.9 & $\mathrm{B}$ & 0.915 & $\mathrm{~T}$ & 45.58 & 33.45 & MgII? & \\
\hline J095701+3207.0 & B & 0.334 & $\mathrm{~F}$ & 43.76 & 32.62 & {$[\mathrm{OII}], \mathrm{H} \delta, \mathrm{H} \gamma, \mathrm{H} \beta,[\mathrm{OIII}], \mathrm{H} \alpha$} & \\
\hline $\mathrm{J} 134252+4032.0^{(2)}$ & $\mathrm{B}$ & 0.909 & $\mathrm{~T}$ & 45.48 & 33.82 & MgII? & \\
\hline $\mathrm{J} 142744+3338.4$ & $\mathrm{~B}$ & 1.237 & $\mathrm{~F}$ & 45.14 & $33.07^{*}$ & $\mathrm{CIII}], \mathrm{MgII}$ & \\
\hline
\end{tabular}

${ }^{a}$ see Table 5 .

${ }^{b}$ see Table 5 .

${ }^{c}$ see Table 5.

Notes:

(1) Also in Lamer et al. (1997) $(z=1.050)$.

(2) Also in Hook et al. (1998) $(z=0.91)$.

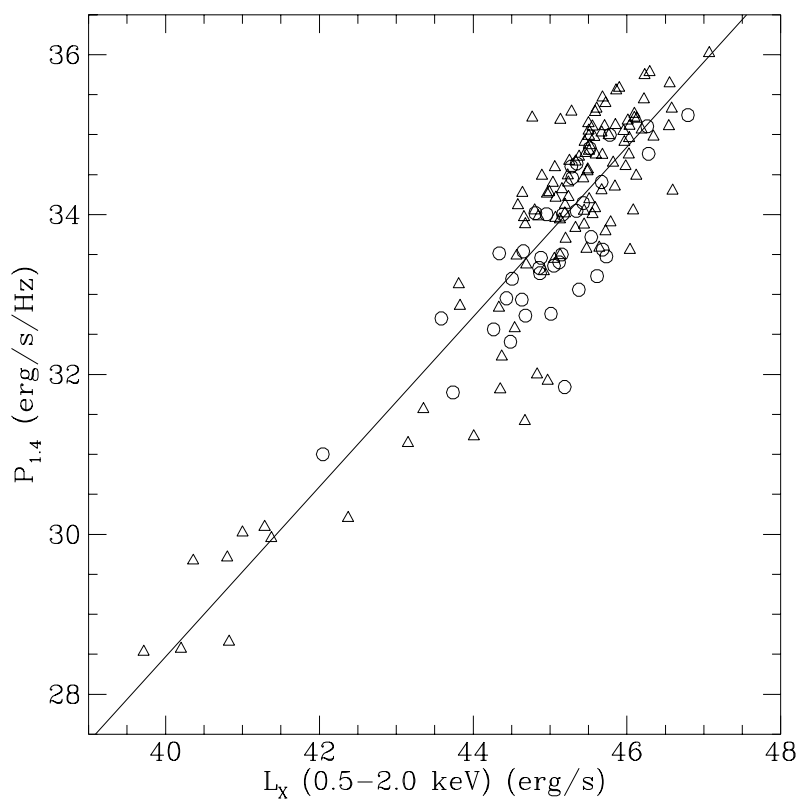

Fig. 5. Monochromatic radio luminosity versus X-ray luminosity for the AGNs belonging to the REX survey and for which we have a measured radio spectral index. The circles represent the objects presented in this paper while the triangles represent the objects previously identified. The solid line represents the best least squares fit to the data

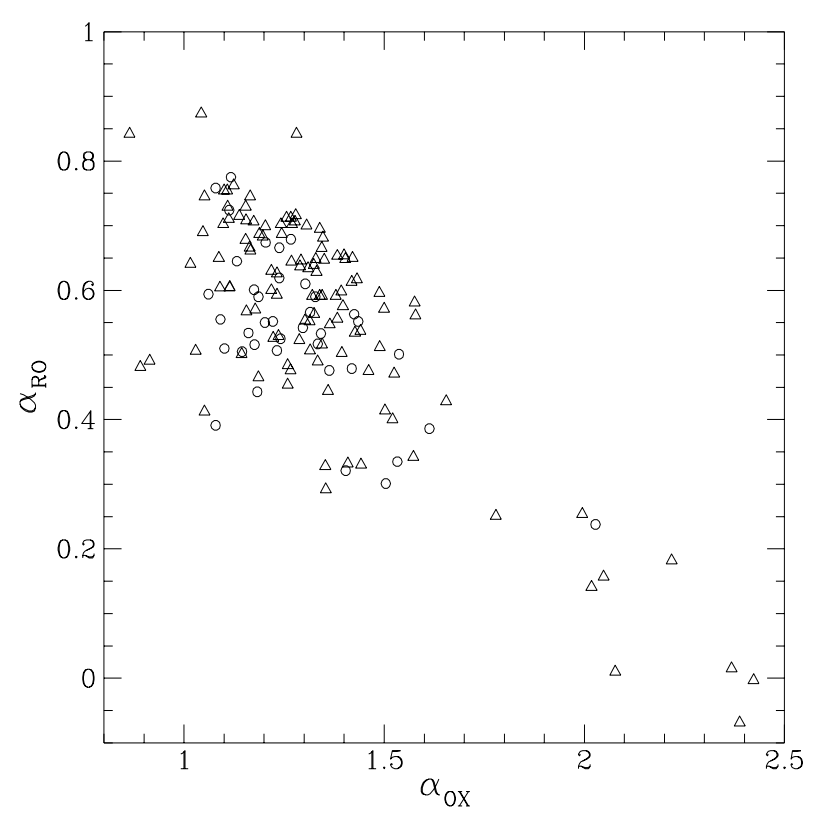

Fig. 6. Radio-optical $\left(\alpha_{\mathrm{RO}}\right)$ vs. the X-ray-optical $\left(\alpha_{\mathrm{OX}}\right)$ spectral indices of the AGN present in the REX survey. Symbols are the same as in Fig. 5 
Space Agency (ASI), by the European Commission, TMR Programme, Research Network Contract ERBFMR XCT960034 "CERES", by the FCT under grant PRO15132/1999 and by the Italian Ministry for University and Research (MURST) under grant Cofin98-02-32. This research has made use of the NASA/IPAC Extragalactic Database (NED) which is operated by the Jet Propulsion Laboratory, California Institute of Technology, under contract with the National Aeronautics and Space Administration.

\section{References}

Brinkmann W., Yuan W., Siebert J., 1997, A\&A 319, 413

Caccianiga A., Maccacaro T., Wolter A., Della Ceca R., Gioia I.M., 1999, ApJ 513, 51

Condon J.J., Cotton W.D., Greisen E.W., et al., 1998, AJ 115, 1693

Corbett E.A., Robinson A., Axon D.J., et al., 1996, MNRAS 281,737

da Costa L.N., Willmer C.N.A., Pellegrini P.S., et al., 1998, AJ 116,1

Della Ceca R., Zamorani G., Maccacaro T., et al., 1994, ApJ 430,533

Dickey J.M., Lockman F.J., 1990, ARA\&A 28, 215

Goncalves A.C., Veron P., Veron-Cetty M.P., 1998, A\&A 333, 877
Gregory P.C., Condon J.J., 1991, ApJS 75, 1011

Griffith M.R., Wright A.E., 1993, AJ 105, 1666

Hill T.L., Heisler C.A., Sutherland R.W., et al., 1999, AJ 117, 111

Hook I.M., Becker R.H., McMahon R.G., White R.L., 1998, MNRAS 297, 1115

Kendall M., Stuart A., 1979, The Advanced Theory of Statistics. MacMillan, New York

Iovino A., Clowes R., Shaver P., 1996, A\&A 119, 265

Lamer G., Brunner H., Staubert R., 1997, A\&A 327, 467

Laurent-Muehleisen S.A., Kollgaard R.L., Ciardullo R., et al., 1998, ApJS 118, 127

Marchã M.J.M., Browne I.W.A., Impey C.D., Smith P.S., 1996, MNRAS 281, 425

Ostebrock D.E., Pogge R., 1985, ApJ 297, 1660

Perlman E., Padovani P., Giommi P., et al., 1998, AJ 115, 1253

Puchnarewicz E.M., Mason K.O., Carrera F., et al., 1997, MNRAS 291, 177

Rizza E., Burns J.O., Ledlow M.J., et al., 1998, MNRAS 301, 328

Rengelink R.B., Tang Y., de Bruyn A.G., et al., 1997, A\&AS 124,259

Stocke J.T., Morris S.L., Gioia I.M., et al., 1991, ApJS 76, 813

Veilleux S., Osterbrock D.E., 1987, ApJS 63, 295

Vermeulen R.C., Ogle P.M., Tran H.D., et al., 1995, ApJ 452, L5

Wolter A., Ruscica C., Caccianiga A., 1998, MNRAS 299, 1047 